\title{
Observation-Based Source Terms in the Third-Generation Wave Model WAVEWATCH III: Updates and Verification ${ }^{\mathscr{O}}$
}

\author{
QINGXiAng LiU, ${ }^{\mathrm{a}}$ W. ERICK Rogers, ${ }^{\mathrm{b}}$ AleXANDER V. BABANIN, ${ }^{\mathrm{a}}$ IAN R. YOUnG, ${ }^{\mathrm{a}}$ \\ LeOnel Romero, ${ }^{c}$ STEFAn ZiEger, ${ }^{\mathrm{d}}$ FANGLi QiaO, ${ }^{\mathrm{e}}$ AND CHANGLONG GuAN ${ }^{\mathrm{f}}$

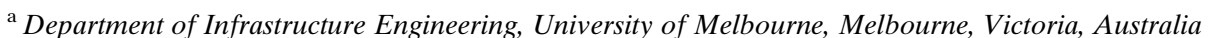 \\ ${ }^{\mathrm{b}}$ Naval Research Laboratory, Stennis Space Center, Mississippi \\ ${ }^{\mathrm{c}}$ Earth Research Institute, University of California, Santa Barbara, Santa Barbara, California \\ ${ }^{\mathrm{d}}$ Bureau of Meteorology, Melbourne, Victoria, Australia \\ ${ }^{\mathrm{e}}$ First Institute of Oceanography, Ministry of Natural Resources, Qingdao, China \\ ${ }^{\mathrm{f}}$ Physical Oceanography Laboratory, Ocean University of China, and Pilot National Laboratory for \\ Marine Science and Technology, Qingdao, China
}

(Manuscript received 27 June 2018, in final form 28 November 2018)

\begin{abstract}
The observation-based source terms available in the third-generation wave model WAVEWATCH III (i.e., the ST6 package for parameterizations of wind input, wave breaking, and swell dissipation terms) are recalibrated and verified against a series of academic and realistic simulations, including the fetch/ duration-limited test, a Lake Michigan hindcast, and a 1-yr global hindcast. The updated ST6 not only performs well in predicting commonly used bulk wave parameters (e.g., significant wave height and wave period) but also yields a clearly improved estimation of high-frequency energy level (in terms of saturation spectrum and mean square slope). In the duration-limited test, we investigate the modeled wave spectrum in a detailed way by introducing spectral metrics for the tail and the peak of the omnidirectional wave spectrum and for the directionality of the two-dimensional frequency-direction spectrum. The omnidirectional frequency spectrum $E(f)$ from the recalibrated ST6 shows a clear transition behavior from a power law of approximately $f^{-4}$ to a power law of about $f^{-5}$, comparable to previous field studies. Different solvers for nonlinear wave interactions are applied with ST6, including the Discrete Interaction Approximation (DIA), the more expensive Generalized Multiple DIA (GMD), and the very expensive exact solutions [using the Webb-Resio-Tracy method (WRT)]. The GMD-simulated $E(f)$ is in excellent agreement with that from WRT. Nonetheless, we find the peak of $E(f)$ modeled by the GMD and WRT appears too narrow. It is also shown that in the 1-yr global hindcast, the DIA-based model overestimates the low-frequency wave energy (wave period $T>16 \mathrm{~s}$ ) by $90 \%$. Such model errors are reduced significantly by the GMD to $\sim 20 \%$.
\end{abstract}

\section{Introduction}

In deep water, the evolution of wind-generated ocean waves can be described by the radiative transfer equation (e.g., Komen et al. 1994; Young 1999):

$$
\frac{d N}{d t}=\frac{S_{\mathrm{in}}\left(+S_{\mathrm{swl}}\right)+S_{\mathrm{ds}}+S_{\mathrm{nl}}+\cdots}{\sigma},
$$

Supplemental information related to this paper is available at the Journals Online website: https://doi.org/10.1175/JPO-D-180137.s1.

Corresponding author: Qingxiang Liu, qingxiang.liu@unimelb. edu.au where $N(k, \theta ; \mathbf{x}, t)=F(k, \theta ; \mathbf{x}, t) / \sigma$ is the wave action density spectrum, $F(k, \theta ; \mathbf{x}, t)$ is the two-dimensional wavenumber spectrum, $\sigma$ is the intrinsic (radian) frequency, $k$ is the wavenumber, and $\theta$ is the propagation direction of wave energy. For deep water the dispersion relation is

$$
\sigma^{2}=g k,
$$

and $g$ is gravitational acceleration. The RHS of (1) represents different physical sources and fluxes of wave energy, including the wind input term $S_{\text {in }}$, wave breaking term $S_{\mathrm{ds}}$, nonlinear wave-wave interaction $S_{\mathrm{nl}}$, and swell decay $S_{\text {swl }}$, among others (Cavaleri et al. 2007; Holthuijsen 2007; Cavaleri et al. 2018). 
The 3-yr field experiment carried out in Lake George, New South Wales, Australia, in 1997-2000 (Young et al. 2005; Donelan et al. 2005) revealed various novel features of wave dynamics. For the wind input $S_{\text {in }}$, the main novel features (Donelan et al. 2006) are the following: 1) $S_{\text {in }}$ is a nonlinear function of the wave spectrum because the growth rate $\gamma$ depends on wave steepness and hence on the spectrum itself; 2) the growth rate $\gamma$ slows down in extreme conditions because of the flow separation (in relative terms; the growth still increases as the wind increases, but not as fast as one would expect by extrapolating the measurement in moderate wind-forcing conditions); and 3 ) wind input doubles over a breaking wave and hence can increase if the breaking rates are substantial (Babanin et al. 2007). For the whitecapping dissipation term $S_{\mathrm{ds}}$, the novel features are as follows: 1) the threshold for inherent wave breaking demonstrates its existence in terms of (significant) wave steepness (Banner et al. 2000; Babanin et al. 2001), and Babanin and Young (2005) established dimensionless value for such threshold across the entire spectrum; 2) the twophase behavior of $S_{\mathrm{ds}}$ is noteworthy: at any frequency the breaking can happen due to inherent reasons, but above the spectral peak the breaking is also enhanced due to the influence of longer waves on shorter ones (Babanin and Young 2005; Young and Babanin 2006); 3 ) the direct dependence of $S_{\mathrm{ds}}$ on the wind speed when $U_{10}$ (the wind speed at $10 \mathrm{~m}$ above the sea surface) exceeds 14-15 $\mathrm{m} \mathrm{s}^{-1}$ (Manasseh et al. 2006); and 4) the directional distribution of $S_{\mathrm{ds}}$ is bimodal rather than isotropic (Young and Babanin 2006).

These Lake George observations resulted in a new set of source functions for wind input $S_{\text {in }}$ (Donelan et al. 2006; Babanin et al. 2007) and whitecapping dissipation $S_{\mathrm{ds}}$ (Babanin and Young 2005; Young and Babanin 2006), which were tested in academic models (Tsagareli et al. 2010; Babanin et al. 2010) and subsequently implemented in Simulating Waves Nearshore (SWAN; Booij et al. 1999) and WAVEWATCH III (WW3; Tolman 1991) by Rogers et al. (2012, hereafter RBW12) and Zieger et al. (2015, hereafter ZBRY15), respectively. Practical modeling also required introduction of further observation-based physics such as swell dissipation $S_{\text {swl }}$ (Babanin 2006, 2011; Young et al. 2013) and negative wind input (ZBRY15; Aijaz et al. 2016; Liu et al. 2017). Once the waves stop breaking, the dissipation continues, but due to a different reason: turbulence production by wave orbital motion (i.e., the so-called swell decay $S_{\text {swl }}$ ) (Babanin 2006; Babanin and Haus 2009; Young et al. 2013). Note that other mechanisms responsible for $S_{\text {swl }}$ based on the interaction of ocean waves and upper ocean turbulence or air turbulence are also available in the literature (e.g., Teixeira and Belcher 2002; Ardhuin and Jenkins 2006; Ardhuin et al. 2009). This complete set of new physics ready for practical forecast and hindcast received the name of ST6 ${ }^{1}$ in 2014 and 2016 public releases of WW3 (WAVEWATCH III Development Group 2016, hereafter T16). Besides, ST6 is now formally part of the SWAN model as well (SWAN Team 2018, version 41.20A). Further academic developments related to ST6 included a new nonlinear interaction term based on the general kinetic equation (Gramstad and Babanin 2016), modules for wave-current interactions (Rapizo et al. 2017), infragravity waves (Nose et al. 2017), and wave-ice interactions (to be released in the 2019 version of WW3).

Since its implementation in SWAN and WW3, this unique source term package, ST6, of $S_{\text {in }}+S_{\mathrm{ds}}+S_{\mathrm{swl}}$ has been proven skillful for different spatial scales and under different weather conditions (e.g., ZBRY15; Aijaz et al. 2016; van Vledder et al. 2016; Liu et al. 2017). ZBRY15 (their Fig. 5) and Stopa et al. (2016, hereafter SABZ16, their Fig. 7), however, also suggested that ST6 was inclined to overestimate the energy level of the high-frequency tail of the spectrum, indicating an inaccurate balance of different source terms in this specific frequency range.

As will be shown in this paper, this shortcoming can be solved by increasing the wind input $S_{\text {in }}$ slightly and then recalibrating tunable parameters of $S_{\mathrm{ds}}$ [i.e., $a_{1}$ and $a_{2}$ in (9) and (10)]. From a practical point of view, a relatively stronger input allows a higher dissipation, which in turn pulls the overestimated spectral tail down to the correct energy level. Through detailed analyses of academic and realistic simulations performed with WW3 (version $5.16^{2}$; T16), we demonstrate that the recalibrated ST6 package not only performs well in predicting commonly used bulk wave parameters (e.g., significant wave height $H_{s}$ and wave periods; appendix A), but also yields a clearly improved estimation of the high-frequency energy level [or specifically, the saturation spectrum $B(f)$ in $(21)$ and mean square slope $\left\langle s^{2}\right\rangle$; appendix A]. Besides, the updated ST6 is also able to produce a realistic transition behavior from $E(f) \cong f^{-4}$ to $E(f) \propto f^{-5}$, where $E(f)$ is the omnidirectional frequency spectrum. ${ }^{3}$

The Discrete Interaction Approximation of $S_{\mathrm{nl}}$ (DIA; Hasselmann et al. 1985) is the crucial component permitting routine application of third-generation wave

\footnotetext{
${ }^{1}$ The third novel feature of $S_{\text {in }}$ and the fourth feature of $S_{\mathrm{ds}}$ are not implemented in ST6 yet.

${ }^{2}$ With minor modification of ST6 codes.

${ }^{3} \propto$ : approximately proportional to.
} 
models (e.g., Hasselmann et al. 1988; Tolman 1991). It however also has some well-known shortcomings as an approximation (see an extended discussion about this issue in section $2 b$ ). To investigate the errors in spectral wave models attributable to the DIA, we first specifically optimize another more accurate nonlinear solver, that is, the Generalized Multiple DIA (GMD; Tolman 2013) for ST6, and then conduct a thorough comparison of model simulations with these two different nonlinear solvers. The most prominent advantage of the GMD-based model over the DIA-based model, as later illustrated in this paper, is that the former shows a much higher accuracy in simulating the energy of long-period waves $(T>16 \mathrm{~s})$. The computational expense of the GMD approach used here, however, is about 5 times larger than that of the DIA.

This paper is organized as follows. Section 2 provides a brief overview of ST6 source functions $\left(S_{\mathrm{in}}+S_{\mathrm{ds}}+\right.$ $\left.S_{\text {swl }}\right)$ and the four-wave resonant interactions $S_{\mathrm{nl}}$. Section 3 describes the updates of ST6 over its predecessor, particularly focusing on the retuning procedure. Section 4 presents a detailed analysis of modeled wave spectra from duration-limited simulations, followed by a thorough validation of model performance with a 1-yr global hindcast in section 5. Conclusions in section 6 finalize this paper.

\section{Parameterizations}

\section{a. ST6 source term package}

A brief overview of the ST6 source terms is given here for completeness. The reader is referred to RBW12 and ZBRY15 and references therein for more details.

\section{1) WIND INPUT $S_{\text {in }}$}

The wind input parameterization $S_{\mathrm{in}}$, formulated by Donelan et al. (2006), is given as follows:

$$
\begin{aligned}
& S_{\text {in }}(k, \theta)=\frac{\rho_{a}}{\rho_{w}} \sigma(k) \gamma(k, \theta) F(k, \theta), \\
& G(k, \theta)=\mu_{1}-\mu_{2}\left\{1+\tanh \left[\mu_{3} \sqrt{B_{n}(k)} W^{2}(k, \theta)-\mu_{4}\right]\right\},
\end{aligned}
$$

and

$$
\gamma(k, \theta)=\alpha_{\text {in }} G(k, \theta) \sqrt{B_{n}(k)} W^{2}(k, \theta),
$$

where $\rho_{a}$ and $\rho_{w}$ are air and water densities, $W(k, \theta)=$ $U_{s} \cos \left(\theta-\theta_{u}\right) / c(k)-1$ is the wind forcing parameter, $U_{s}$ is the scaling wind speed, $c(k)=\sigma / k$ is the phase velocity, and $B_{n}(k)=B(k) A(k)$ is a spectral measure of wave steepness-the saturation spectrum $B(k)=k^{3} F(k)$
(Phillips 1984) normalized by the spreading function $A$ [O(1); Babanin and Soloviev 1998b]. Also, $G(k, \theta)$ represents the degree of flow separation (whether the full separation occurs or not) and $\alpha_{\text {in }}$ is the wind growth parameter:

$$
\alpha_{\text {in }}=\left\{\begin{array}{cl}
1, & \text { for } \quad W(k, \theta) \geq 0 \\
-a_{0}, & \text { for } \quad W(k, \theta)<0
\end{array}\right.
$$

Here $a_{0}$ is a tuning parameter controlling the strength of negative wind input (ZBRY15; Liu et al. 2017, and references therein). The specific values of the four parameters $\mu_{i}, i=1, \ldots, 4$ in (4) vary with the scaling wind speed $U_{s}$ adopted in $W(k, \theta)$. For $U_{s}=U_{10}$, Donelan et al. (2006) suggested

$$
\mu_{1}=2.8, \quad \mu_{2}=1.0, \quad \mu_{3}=10, \quad \text { and } \quad \mu_{4}=11 .
$$

The wave model community, however, prefers $u_{*}$ scaling in order to assure a consistent fetch law across different wind speeds (Komen et al. 1994, p. 253), where $u_{*}$ is the friction velocity. Therefore, RBW12 advocated using an approximation

$$
U_{s}=U_{10} \simeq \Upsilon u_{*}, \quad \text { and } \quad \Upsilon=28
$$

by following Komen et al. (1984).

\section{2) WAVE BREAKING $S_{\mathrm{ds}}$}

The wave breaking parameterization $S_{\mathrm{ds}}$ of the ST6 package incorporates two different mechanisms: 1) the inherent wave breaking $T_{1}(k, \theta)$ occurring at each frequency once the steepness of that wave component exceeds a threshold value (Banner et al. 2000; Babanin et al. 2001) and 2) the induced breaking of relatively short waves $T_{2}(k, \theta)$ due to the modulation of longer waves (Donelan 2001; Young and Babanin 2006). The source term reads (RBW12; ZBRY15)

$$
\begin{aligned}
& S_{\mathrm{ds}}(k, \theta)=T_{1}(k, \theta)+T_{2}(k, \theta), \\
& T_{1}(k, \theta)=-a_{1} f\left[\frac{\Delta(k)}{F_{T}(k)}\right]^{p_{1}} F(k, \theta), \quad \text { and } \\
& T_{2}(k, \theta)=-a_{2} \int_{f_{\min }}^{f}\left[\frac{\Delta(k)}{F_{T}(k)}\right]^{p_{2}} d f F(k, \theta),
\end{aligned}
$$

where $a_{1}, a_{2}, p_{1}$, and $p_{2}$ are tunable parameters, $f_{\min }$ is the lowest discrete frequency defined in the spectral grid, $F_{T}(k)=B_{T} / k^{3}$ is the spectral threshold, $B_{T}=$ $0.035^{2}$ is the dimensionless saturation-threshold value, and $\Delta(k)=F(k)-F_{T}(k)$ is the exceedance level (Babanin et al. 2010). RBW12 found that highly nonlinear $T_{1}$ and $T_{2}$ are required to balance the strong wind 
input [(3)] beyond the spectral peak. This was achieved by setting $p_{1}=p_{2}=4$.

\section{3) Swell disSipation $S_{\text {swl }}$}

The swell dissipation term $S_{\text {swl }}$ of ST6 characterizes the loss of wave energy as a result of the turbulence production by nonbreaking surface waves (Babanin 2006; Babanin and Haus 2009). According to Babanin (2011) and Young et al. (2013), ZBRY15 implemented $S_{\text {swl }}$ as

$$
S_{\text {swl }}(k, \theta)=-\frac{2}{3} b_{1} \sigma(k) \sqrt{B_{n}(k)} F(k, \theta),
$$

where the dimensionless proportionality coefficient $b_{1}$ is hypothesized to be steepness-dependent in the following way:

$$
b_{1}=\frac{B_{1} H_{s} k_{p}}{2} .
$$

Here $k_{p}$ is the peak wavenumber and $B_{1}$ is a tunable scaling coefficient. Note that $S_{\text {swl }}$ is applied to both wind sea and swell. But in the context of wind sea, it does not contribute significantly to the source term balance (e.g., ZBRY15).

\section{b. Nonlinear wave-wave interactions $S_{\mathrm{nl}}$}

Hasselmann (1962) pointed out that four wave components satisfying the resonance condition

$$
\left\{\begin{array}{c}
\mathbf{k}_{1}+\mathbf{k}_{2}=\mathbf{k}_{3}+\mathbf{k}_{4}, \\
\sigma_{1}+\sigma_{2}=\sigma_{3}+\sigma_{4}
\end{array},\right.
$$

could exchange energy and momentum, where $\mathbf{k}$ is the wavenumber vector. The set of these four waves is also known as a quadruplet. The nonlinear term $S_{\mathrm{nl}}(k, \theta)$ describes the redistribution of wave energy over the spectrum resulted from such resonant wave-wave interactions. The important role of $S_{\mathrm{nl}}$ in the evolution of wave spectrum is well founded (e.g., Hasselmann et al. 1973; Young and van Vledder 1993). The computation of its exact solutions, such as by the Webb-Resio-Tracy (WRT) method (Webb 1978; Tracy and Resio 1982; Resio and Perrie 1991; van Vledder 2006), however, is extremely time-consuming, and this prohibits its applicability to large-scale wave hindcasting and forecasting. Hasselmann et al. (1985) proposed the DIA approach to overcome this difficulty by accounting for interaction contributions for a single representative quadruplet only, defined by (13) and

$$
\left\{\begin{array}{l}
\mathbf{k}_{1}=\mathbf{k}_{2} \\
\sigma_{3}=(1+\lambda) \sigma_{1}, \\
\sigma_{4}=(1-\lambda) \sigma_{1}
\end{array}\right.
$$

where $\lambda$ is a free shape parameter. The DIA is not only several orders of magnitude more efficient (e.g., Tolman 2013, his Table 4), but also retains the dominant features of the exact solutions (Hasselmann et al. 1985; Young et al. 1987). Accordingly, it has been widely used for decades in third-generation spectral wave models (Hasselmann et al. 1988; Tolman 1991; Booij et al. 1999).

Nonetheless, the shortcomings of DIA have also been extensively discussed. First, the two lobes of $S_{\mathrm{nl}}$ beyond the peak frequency $f_{p}$-one negative lobe close to $f_{p}$ and one positive lobe at higher frequenciescomputed by the DIA are clearly different from the exact solutions (Hasselmann et al. 1985; Cavaleri et al. 2007). As a result, the DIA may not be able to yield a correct form of the equilibrium range $\left(f^{-4}\right)$ of the wind wave spectra (Resio et al. 2016). Second, wave spectra from the DIA are too broad in both frequency and directional space (e.g., Young et al. 1987; Cavaleri et al. 2007; Rogers and van Vledder 2013). Third, the DIA could fail to reproduce the directional bimodality of short waves because of its tendency to misplace the two lobes of $S_{\mathrm{nl}}$ beyond $f_{p}$ (van der Westhuysen et al. 2007; Cavaleri et al. 2007). Finally, it is also noteworthy that under hurricane conditions the DIA may give rise to $\sim 20 \%$ errors in the simulated $H_{s}$, as shown in Tolman (2013) and Liu et al. (2017). Liu et al. (2017, their Fig. 13) also demonstrated that for cross swell the low-frequency wave energy simulated by DIA is clearly higher than that by WRT.

Tolman (2013) developed a more accurate parameterization of $S_{\text {nl }}$ (i.e., the GMD) by expanding the DIA in several ways. The definition of the representative quadruplet [(14)] is extended as follows:

$$
\left\{\begin{array}{l}
\sigma_{2}=\frac{1-\mu}{1+\mu} \sigma_{1} \\
\sigma_{3}=\frac{1+\lambda}{1+\mu} \sigma_{1} \\
\sigma_{4}=\frac{1-\lambda}{1+\mu} \sigma_{1} \\
\theta_{12}=\arccos \frac{\mathbf{k}_{1} \cdot \mathbf{k}_{2}}{k_{1} k_{2}}
\end{array}\right.
$$

where $\mu$ is the second shape parameter and $\theta_{12}$ is the angle between $\mathbf{k}_{1}$ and $\mathbf{k}_{2}$. Clearly, (14) is a simplified form of (15) for $\mu=0$ and $\theta_{12}=0^{\circ}$. Another important feature of the GMD is its capacity of incorporating interaction contributions for multiple representative quadruplets, rather than one quadruplet only by the DIA. For brevity, other advantageous features of the GMD over the DIA are not described here. The reader is referred to Tolman (2010), Tolman (2013), and 
Tolman and Grumbine (2013) for further details. These three studies showed that the accuracy of the GMD increases with increasing numbers of free parameters used in (15) [i.e., one-parameter $(\lambda)$, two-parameter $(\lambda, \mu)$, and three-parameter $\left(\lambda, \mu, \theta_{12}\right)$ quadruplet layout] and increasing numbers of quadruplets $n_{q}$. For the threeparameter $\left(\lambda, \mu, \theta_{12}\right)$ quadruplet definition, the improvement of GMD accuracy will saturate at $n_{q}=5$ or 6 . Considering this, we chose the GMD configuration with five quadruplets and a three-parameter quadruplet definition $^{4}$ in our following analysis. For convenience, we will also simply refer to this specific GMD configuration as GMD. Figure 1, as an example, clearly illustrates the differences in quadruplet layouts used by the DIA (Fig. 1a) and the GMD (Fig. 1b). Note that only the deep scaling function of the GMD is used in our manuscript, which represents "weak" four-wave nonlinear interactions in deep water and intermediate depths for $k d \geq 0.75$ ( $d$ is water depth; Tolman 2013). This is comparable to the applicable range of the DIA and its $k d$-dependent scaling relation for $k d \geq 0.8$ (e.g., Hasselmann and Hasselmann 1985; Hasselmann et al. 1988). The application of ST6 with other nonlinear solvers such as the simplified Research Institute for Applied Mechanics (RIAM; Komatsu and Masuda 1996; Tamura et al. 2008) and two-scale approximation (TSA; Resio and Perrie 2008; Perrie et al. 2013) is beyond the scope of this paper, and therefore may be pursued in the future.

\section{Model calibrations}

Herein ST6 is applied with different parameterizations of $S_{\mathrm{n}}$. To distinguish these model configurations, we will refer to ST6 + DIA as $\mathrm{ST}^{D}$, ST6 + GMD as $\mathrm{ST}^{G}$, and ST6 + WRT as ST6 ${ }^{W}$. When necessary, the combination of the ST4 source terms (Ardhuin et al. 2010) and DIA (hereafter $\mathrm{ST}^{D}$ ), which is used for the operational forecasting in NOAA's National Centers for Environmental Prediction (NCEP; Alves et al. 2015), is also included for comparisons. The wind growth parameter $\beta_{\max }$ of ST4 ${ }^{D}$ used is 1.33 (Ardhuin et al. 2010; Rascle and Ardhuin 2013), unless otherwise specified.

\section{a. Calibration of $S T 6^{D}$}

As mentioned in section 1, our (re)calibration of $\mathrm{ST} 6^{D}$ is conducted by increasing the (positive) wind input term slightly, and then finding the new tunable parameters existing in the wave breaking and swell decay terms (i.e., $a_{0}, a_{1}, a_{2}, B_{1}$ ). The amplification of $S_{\text {in }}$ is

\footnotetext{
${ }^{4}$ The G35d configuration in Tolman (2013).
}
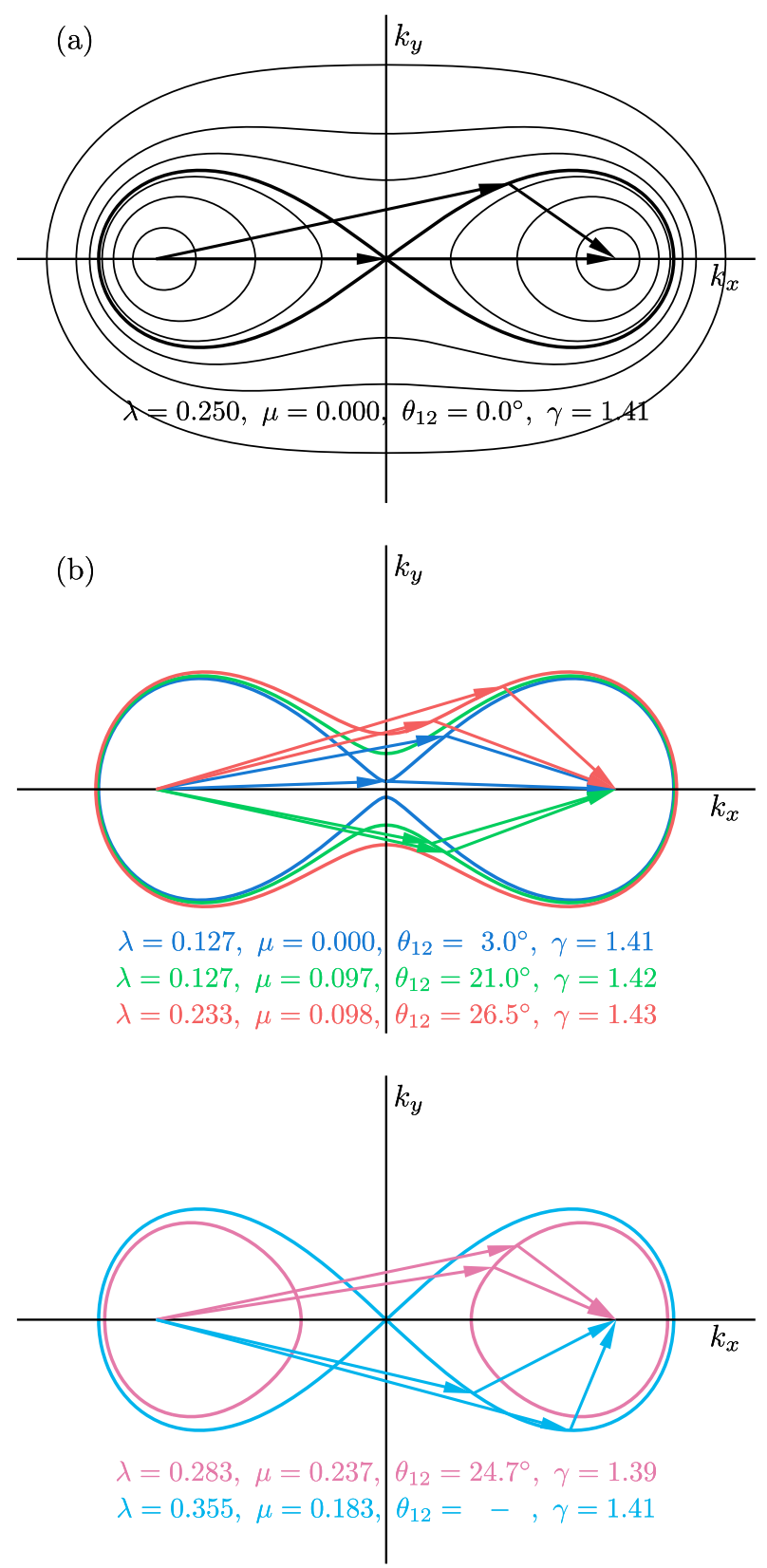

FIG. 1. Interaction diagram for $\mathbf{k}_{1}+\mathbf{k}_{2}=\mathbf{k}_{3}+\mathbf{k}_{4}=\mathbf{k}$ [after Hasselmann (1963)] with solid lines for contours of $\gamma=$ $\left(\sqrt{k_{1}}+\sqrt{k_{2}}\right) / \sqrt{\|\mathbf{k}\|}$ and vectors for quadruplet layouts: (a) the DIA quadruplet and (b) the five GMD quadruplets as specified in Table 1 . For the given values of $\left(\lambda, \mu, \theta_{12}\right)$, only one solution of (15) is shown for the clarity of this figure.

achieved by setting $U_{s} \simeq \Upsilon u_{*}=32 u_{*}$, as compared with $\mathrm{Y}=28$ in (7). It was found by coauthor W. Erick Rogers (2014, unpublished work) that using $\Upsilon=32$ could improve model skills in estimating tail level in the ST6 implementation in SWAN [see also Rogers (2017)]. RBW12 calibrated $a_{1}$ and $a_{2}$ [see (9) and (10)] by using a 
single-grid-point, duration-limited simulation. Because of the scarcity of observations in such idealized cases, the authors had to adopt growth curves simulated by other widely used packages (Komen et al. 1984; Rogers et al. 2003) as references. Unlike RBW12, here we decided to tune the model with fetch-limited simulations, particularly considering the extensive field studies of fetch-limited wind wave spectra (e.g., Hasselmann et al. 1973; Kahma and Calkoen 1992; Babanin and Soloviev 1998a; Romero and Melville 2010a, hereafter RM10).

For tuning purposes, we utilized a fetch-limited test under a homogeneous wind forcing with $U_{10}=20 \mathrm{~m} \mathrm{~s}^{-1}$, blowing perpendicularly to a straight shoreline. Similar to Tolman and Chalikov (1996), we employed three 40-point grids with different spatial resolutions $(\Delta x=$ $2.5,25$, and $250 \mathrm{~km}$, respectively) to guarantee a wide range of fetches. The spectral grid was discretized as $\Delta f / f=0.07$ and $\Delta \theta=10^{\circ}$, with $f_{0} \in[0.037,1.027] \mathrm{Hz}$, $i=1, \ldots, 50$. The fetch law for stable stratification, as suggested by Kahma and Calkoen (1992, hereafter KC92) (see also Komen et al. 1994, p. 181), was selected as the tuning reference:

$$
\left\{\begin{array}{l}
\varepsilon_{*}=\frac{H_{s}^{2} g^{2}}{16 u_{*}^{4}}=2.1 \times 10^{-3} \chi_{*}^{0.79} \\
\nu_{*}=\frac{f_{p} u_{*}}{g}=2.3 \chi_{*}^{-0.25} / 2 \pi
\end{array}\right.
$$

where $\chi_{*}=g X / u_{*}^{2}, X$ is the fetch, and $f_{p}$ is the peak frequency. It is interesting to note that the fetch law from Babanin and Soloviev (1998a) is remarkably consistent with (16), except for $\nu_{*}$ at short fetches (Fig. 2). The power law suggested by RM10 is also in excellent agreement with the former two studies, particularly for the dimensionless energy $\varepsilon_{*}$.

We first made an attempt to determine $\left(a_{1}, a_{2}\right)$ for $\mathrm{ST} 6^{D}$ by the following subjective, loose rules:

1) $\mathrm{ST}^{D}{ }^{D}$-simulated dimensionless energy $\varepsilon_{*}$ and peak frequency $\nu_{*}$ should match the KC92 growth curves in (16) reasonably well. Specifically, $b_{n}^{\varepsilon *} \geq 0, \epsilon_{n}^{\varepsilon *} \leq$ $40 \%$, and $\epsilon_{n}{ }^{*} \leq 10 \%$, where $b_{n}$ and $\epsilon_{n}$ are the normalized bias and root-mean-square error (RMSE; appendix A). The positiveness of $b_{n}^{\varepsilon *}$ is imposed due to the exclusion of the negative $S_{\text {in }}$ and $S_{\mathrm{swl}}$ terms at this stage (i.e., $a_{0}=B_{1}=0$ ). ${ }^{5}$

\footnotetext{
${ }^{5}$ According to our tuning exercises, $\epsilon_{n}^{\nu *}$ is remarkably less sensitive to the swell dissipation (i.e., negative $S_{\text {in }}$ and $S_{\text {swl }}$ ) than $\epsilon_{n}^{\varepsilon *}$. Such swell-related dissipation terms may result in $\sim 10 \%$ differences in $\epsilon_{n}^{\varepsilon *}$. Therefore, the criterion for $\epsilon_{n}^{\varepsilon *}$ was only loosely imposed (e.g., $\left.\epsilon_{n}^{\varepsilon *} \leq 40 \%\right)$.
}
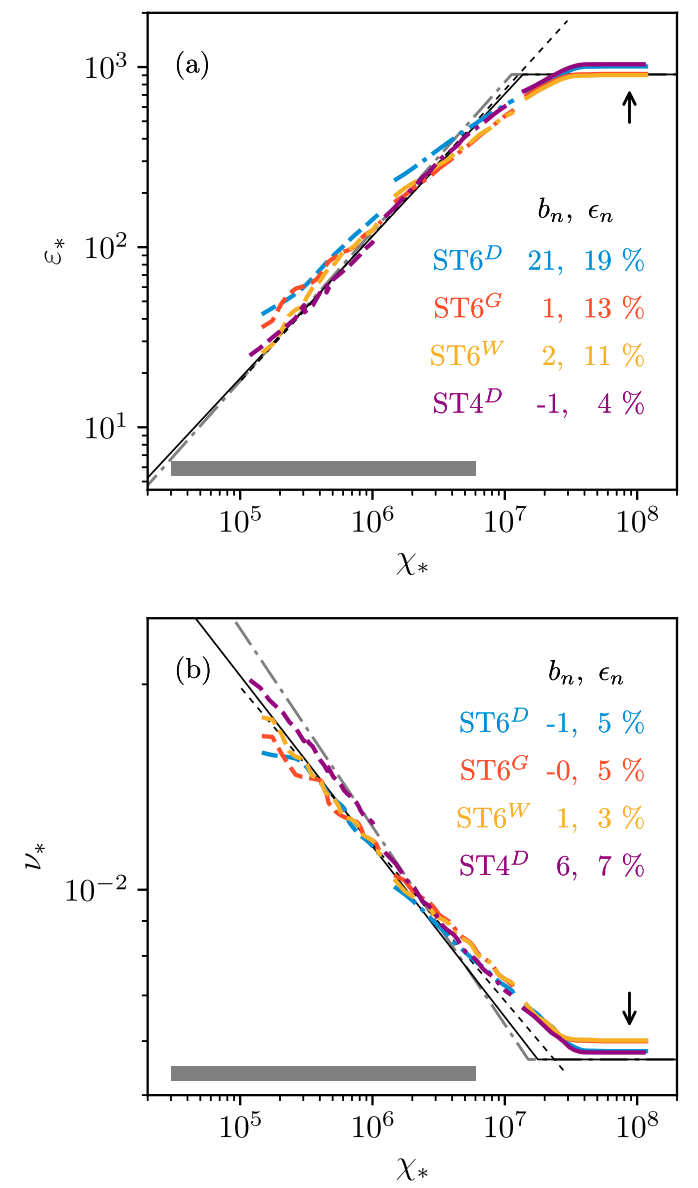

FIG. 2. (a) Dimensionless energy $\varepsilon_{*}=H_{s}^{2} g^{2} / 16 u_{*}^{4}$ and (b) peak frequency $\nu_{*}=f_{p} u_{*} / g$ as a function of dimensionless fetch $\chi_{*}=g X / u_{*}^{2}$. The solid black lines represent the KC92 growth curves [(16)] and the Pierson-Moskowitz asymptotic limits [(17)]. The gray dash-dotted lines and black dotted lines are growth curves from Babanin and Soloviev (1998a) and Romero and Melville (2010a), respectively. Results from different model configurations (1D runs; see section 3c) after a 72-h model run are color-coded by blue for $\mathrm{ST}_{6}{ }^{D}$, red for $\mathrm{ST}_{6}{ }^{G}$, yellow for $\mathrm{ST} 6^{W}$, and purple for $\mathrm{ST} 4^{D}$. The corresponding overall error metrics (normalized bias $b_{n}$ and RMSE $\epsilon_{n}$; see appendix A) are also printed. Only model points within the valid range of KC92 growth curves (highlighted by the thick gray horizontal lines) were included in error analyses. Besides, the first five points of each grid were abandoned to reduce the effects of numerical errors (Tolman 1992). The dashed, dash-dotted, and solid colored lines denote points from grids with the resolution of $\Delta x=2.5,25$, and $250 \mathrm{~km}$, respectively. The black arrows indicate the point where $X=7500 \mathrm{~km}$, which was chosen to compute $\int T_{2} d f / \int\left(T_{1}+T_{2}\right) d f$ for fully developed waves. Note that the negative $S_{\text {in }}$ and $S_{\text {swl }}$ are activated here.

2) For fully developed waves, for example, waves at the 30th point of the coarsest grid where $X=7500 \mathrm{~km}$ (black arrows in Fig. 2), the two-phase wave breaking term $S_{\mathrm{ds}}$ in (8) should satisfy $75 \% \leq \int T_{2} d f /$ $\int\left(T_{1}+T_{2}\right) d f \leq 80 \%$ (RBW12). 
TABLE 1. Parameter setting for $\mathrm{ST}^{D}{ }^{D}, \mathrm{ST} 6^{G}$, and $\mathrm{ST} 6^{W}$, including five parameters $\left(\Upsilon, a_{0}, a_{1}, a_{2}, B_{1}\right)$ from the ST6 source terms $\left(S_{\text {in }}\right.$, $\left.S_{\mathrm{ds}}, S_{\mathrm{swl}}\right)$, and four parameters $\left(\lambda, \mu, \theta_{12}, C_{q}\right)$ pertaining to the DIA-like (i.e., DIA and GMD) parameterizations of $S_{\mathrm{nl}}$, where $C_{q}$ is a proportionality constant [ $C$ in Hasselmann et al. (1985) and $C_{\text {deep }}$ in Tolman (2013)].

\begin{tabular}{|c|c|c|c|c|c|c|c|c|c|}
\hline & $\Upsilon$ & $a_{0}$ & $a_{1}$ & $a_{2}$ & $B_{1}$ & $\lambda$ & $\mu$ & $\theta_{12}\left({ }^{\circ}\right)$ & $C_{q}$ \\
\hline $\mathrm{ST}^{D}$ & 32.0 & 0.09 & $4.75 \times 10^{-6}$ & $7.00 \times 10^{-5}$ & $4.10 \times 10^{-3}$ & 0.25 & - & - & $3.00 \times 10^{7}$ \\
\hline \multirow[t]{5}{*}{$\mathrm{ST}^{G}$} & 32.0 & 0.05 & $4.75 \times 10^{-6}$ & $7.00 \times 10^{-5}$ & $6.00 \times 10^{-3}$ & 0.127 & 0.000 & 3.0 & $4.88 \times 10^{7}$ \\
\hline & & & & & & 0.127 & 0.097 & 21.0 & $1.26 \times 10^{8}$ \\
\hline & & & & & & 0.233 & 0.098 & 26.5 & $6.20 \times 10^{7}$ \\
\hline & & & & & & 0.283 & 0.237 & 24.7 & $2.83 \times 10^{7}$ \\
\hline & & & & & & 0.355 & 0.183 & ${ }^{\mathrm{a}}$ & $1.17 \times 10^{7}$ \\
\hline ST $6^{W}$ & 32.0 & 0.05 & $4.75 \times 10^{-6}$ & $7.00 \times 10^{-5}$ & $6.00 \times 10^{-3}$ & - & - & - & - \\
\hline
\end{tabular}

${ }^{\mathrm{a}}$ For the fifth quadruplet layout of GMD (light blue arrows in Fig. 1), the three-parameter $\left(\lambda, \mu, \theta_{12}\right)$ definition [(15)] degrades to a twoparameter $(\lambda, \mu)$ form, and $\theta_{12}$ is implied by the value of $\mu$ (Tolman 2013). Accordingly, a combination of ST6 ${ }^{G}$ and the conservative nonlinear high-frequency filter of Tolman (2011) might be necessary to stabilize the model integration, particularly for high-resolution spectral grid (say, $\Delta \theta \leq 5^{\circ}$ ).

Through our tuning exercises, we found that the two restrictions described above yield a narrow corridor in the $\left(a_{1}, a_{2}\right)$ parameter space [see section 1 of the supplemental online material (SOM)]. To further refine these two parameters, we added another important supplementary constraint (rule 3, below).

3) For a realistic 75-day wave hindcast in Lake Michigan, ${ }^{6} \mathrm{ST} 6^{D}$ should predict both $H_{s}$ and mean square slope $\left\langle s^{2}\right\rangle$ quite accurately (e.g., the RMSE $\epsilon$ of $H_{s}$ and $\left\langle s^{2}\right\rangle$ are less than $0.2 \mathrm{~m}$ and $10^{-3}$, respectively), as compared with measurements from a single buoy 45007 (see section 2 of the SOM).

Waves prevailing in Lake Michigan are generally young and free of wind-swell interactions (Rogers and van Vledder 2013). Accordingly, the deactivation of $S_{\text {swl }}$ and negative $S_{\text {in }}$ in this hindcast experiment is still physically sound.

After the optimal $\left(a_{1}, a_{2}\right)$ for $\mathrm{ST}^{D}{ }^{D}$ was established by the above-mentioned approach $\left(a_{1}=4.75 \times 10^{-6}, a_{2}=\right.$ $7.00 \times 10^{-5}$; Table 1$)$, we continued the calibration of the negative wind input parameter $a_{0}$ in (6) and the swell decay coefficient $B_{1}$ in (12). Following ZBRY15, these two parameters were determined through a global hindcast of the year 2013, using a coarse longitude-latitude grid $\left(1.25^{\circ} \times 1.0^{\circ}\right)$ forced by winds from the NCEP Climate Forecast System version 2 (hereafter CFSv2; Saha et al. 2014). ${ }^{7}$ By comparing with measurements from the cross-calibrated altimeter dataset produced by Young et al. (2017), we found that ST $6^{D}$ with $a_{0}=0.09$ and $B_{1}=4.10 \times 10^{-3}$ (Table 1$)$ provided acceptable skills in specification of $H_{s}$ in

\footnotetext{
${ }^{6}$ The model attributes are detailed in Rogers and Wang (2007) and ZBRY15.

${ }^{7}$ Data sourced from NCAR's Research Data Archive (https:// rda.ucar.edu/datasets/ds094.0).
}

global basins (section 3 of the SOM; bias $b=0.03 \mathrm{~m}$ and RMSE $\epsilon=0.39 \mathrm{~m}$ ). Thus far, this finalizes the entire calibration procedure of $\mathrm{ST}^{D}$.

Figure 2 shows the $\mathrm{ST}^{D}$-simulated dimensionless energy $\varepsilon_{*}$ and peak frequency $\nu_{*}$ as a function of dimensionless fetch $\chi_{*}$ in the fetch-limited test under $U_{10}=20 \mathrm{~m} \mathrm{~s}^{-1}$. In general, wave parameters given by ST $^{D}$ are in reasonable agreement with KC92 curves in (16), as forced in our tuning process. Within the valid range of the fetch-limited observations from KC92 $\left(3 \times 10^{4} \leq \chi_{*} \leq 6 \times 10^{6}\right.$; see Komen et al. 1994, p. 180 , RM10), ${ }^{8}$ the overall $\epsilon_{n}$ of $\varepsilon_{*}$ and $\nu_{*}$ are $19 \%$ and $5 \%$, respectively. At short and intermediate fetches (e.g., $\left.\chi_{*}<6 \times 10^{6}\right), \mathrm{ST}^{D}$ overestimates $\varepsilon_{*}$ moderately with an overall $b_{n}$ of $21 \%$. At extremely long fetches, $\mathrm{ST}^{D}$ agrees quite well with the Pierson-Moskowitz (PM) asymptotic limits, given by

$$
\left\{\begin{array}{l}
\varepsilon_{*}^{\mathrm{PM}}=\varepsilon_{10}^{\mathrm{PM}} /\left.C_{d}^{2}\right|_{U_{10}=20 \mathrm{~m} \mathrm{~s}^{-1}}=0.91 \times 10^{3} \\
\nu_{*}^{\mathrm{PM}}=\left.\nu_{10}^{\mathrm{PM}} \sqrt{C_{d}}\right|_{U_{10}=20 \mathrm{~m} \mathrm{~s}^{-1}}=5.64 \times 10^{-3}
\end{array},\right.
$$

where $\varepsilon_{10}^{\mathrm{PM}}$ and $\nu_{10}^{\mathrm{PM}}$ are the $U_{10}$-scaled PM limits from Alves et al. [2003, their (17)] and $C_{d}$ is the winddependent drag coefficient. Here we took $C_{d}=$ $2.1 \times 10^{-3}$ for $U_{10}=20 \mathrm{~m} \mathrm{~s}^{-1}$ by following the drag law described in Hwang (2011), which is also the drag law utilized by ST6 to estimate $u_{*}$ (RBW12). In this case $\mathrm{ST} 4^{D}$ presents an improved accuracy for $\varepsilon_{*}\left(\epsilon_{n}^{\varepsilon *}=4 \%\right)$ and a slightly degraded accuracy for $\nu_{*}\left(\epsilon_{n}^{\nu^{*}}=7 \%\right)$. The fully developed sea states reached by $\mathrm{ST} 4^{D}$

\footnotetext{
${ }^{8}$ Komen et al. (1994, p. 180) shows that the valid range of the KC92 fetch-limited measurements is $\chi_{*} \in\left[3 \times 10^{4}, 4 \times 10^{6}\right]$. According to Romero and Melville (2010a, their Figs. 7c,d), the upper limit of the valid range can be safely extended to $\chi_{*}=6 \times 10^{6}$.
} 
are very close to those for $\mathrm{ST}^{D}\left(\varepsilon_{*} \simeq 1.00 \times 10^{3}\right.$, $\left.\nu_{*} \simeq 5.8 \times 10^{-3}\right)$.

\section{b. Calibration of $\mathrm{ST6}^{G}$}

For the GMD parameterization of $S_{\mathrm{nl}}$ with five quadruplets and a three-parameter $\left(\lambda, \mu, \theta_{12}\right)$ quadruplet definition [(15)], there are a total of 20 free parameters to be determined. ${ }^{9}$ Tolman and Grumbine (2013) designed a holistic genetic optimization (GO) technique to efficiently optimize these parameters altogether. The five quadruplet layouts,${ }^{10}$ yielded by the GO algorithm specifically for the ST6 package, are presented in Table 1 and Fig. 1. For idealized test cases selected in Tolman and Grumbine (2013) and relative to the WRT, such a GMD configuration reduces errors of the DIA by more than $50 \%$ (section 4 of the SOM), consistent with the findings in Tolman (2013).

Using the same $\left(a_{1}, a_{2}\right)$ from $\mathrm{ST}^{D}{ }, \mathrm{ST}^{G}$ shows approximately $20 \%$ lower $\varepsilon_{*}$ and $1 \%$ higher $\nu_{*}$ with slightly improved $\epsilon_{n}^{\varepsilon *}(13 \%)$ and unchanged $\epsilon_{n}^{\nu *}(5 \%)$ in the fetch-limited test (Fig. 2). The overestimation of $\varepsilon_{*}$ at intermediate fetches by $\mathrm{ST}^{D}$ is noticeably improved by $\mathrm{ST}^{G}{ }^{G}$. Considering that all three tuning rules described in the previous section are still well satisfied, we decided not to adjust $\left(a_{1}, a_{2}\right)$ for ST $6^{G} \cdot{ }^{11}$ By contrast, the global hindcast experiment for 2013 suggested an update of $\left(a_{0}, B_{1}\right)$ to $\left(0.05,6.00 \times 10^{-3}\right)$ in $\mathrm{ST}^{G}$ (Table 1) is useful to further enhance model accuracy of $H_{s}$ (section 3 of the SOM).

Since it is extremely expensive, if not impossible, to run $\mathrm{ST}^{W}{ }^{W}$ in realistic large-scale applications, we did not try a further calibration of ST6 ${ }^{W}$. For simplicity, ST $6^{W}$ directly inherits all the parameters (i.e., $a_{0}, a_{1}$, $\left.a_{2}, B_{1}\right)$ from $\mathrm{ST}^{G}$, as shown in Table 1. Examination of Fig. 2 demonstrates that ST6 ${ }^{W}$ conforms very well to ST6 ${ }^{G}$, except at short fetches $\left(\chi_{*}<4 \times 10^{5}\right)$. Among the three ST6 model configurations, ST $6^{W}$ produces the lowest errors with $\epsilon_{n}^{\varepsilon *}=11 \%$ and $\epsilon_{n}^{\nu *}=3 \%$. The

\footnotetext{
${ }^{9}$ For each quadruplet, a proportionality constant $C_{q}$, which controls the magnitude of interactive contributions for this given quadruplet [ $C_{\text {deep }}$ in Tolman 2013, his (24)], is also freely tunable in the GMD parameterization.

${ }^{10}$ This GMD configuration, different from the G35d configuration in Tolman (2013, his Table 2), is specifically optimized for ST6. The optimization experiments should be repeated for other source term packages (see Tolman 2013, and section 4 of our SOM).

${ }^{11}$ It, however, may be worth mentioning that in the Lake Michigan hindcast, $\mathrm{ST}^{G}$ produces somewhat inferior skills to those for $\mathrm{ST}^{D}$ in specification of $H_{s}$ and wave periods (Table S1 of the SOM). Similarly, Rogers and van Vledder (2013) also reported that the DIA-based model runs perform better for these two wave parameters than the WRT-based runs in their Lake Michigan simulations.
}

asymptotic values for $\nu_{*}$ from $\mathrm{ST}^{G}{ }^{G}$ and $\mathrm{ST} 6^{W}$ are slightly higher than the value suggested by Alves et al. (2003) $\left(6.00 \times 10^{-3}\right.$ vs $\left.5.64 \times 10^{-3}\right)$.

\section{c. Fetch geometry}

The last topic we would like to discuss in this section is the effect of fetch geometry on wave growth (Young 1999, p. 109). Field studies (Pettersson and Kahma 2005; Ataktürk and Katsaros 1999) suggested that for the same dimensionless fetch $\chi$, the dimensionless energy $\varepsilon$ values of mature waves were remarkably lower for the narrow fetch than for the broad fetch. The dimensionless frequency $\nu$ was also affected to some extent, but was clearly less sensitive than $\varepsilon$. It was believed that the narrow geometry constrained the development of waves propagating along directions oblique to the long axis of narrow fetches (bays or lakes). As demonstrated in Rogers and Wang (2006, their Fig. 15) and Ataktürk and Katsaros (1999, p. 643), the thirdgeneration wave models are able to provide qualitatively consistent behavior. An interesting detail, which we think is worth mentioning and which we found from our simulations, is that the DIA-based results are more sensitive to the fetch geometry than those from the GMD and WRT.

Figure 3 presents the fetch-limited results from the one-dimensional (1D) run (i.e., the propagation of wave energy along the $y$ direction was switched off, and the model domain became essentially infinitely wide) and the two-dimensional (2D) run (i.e., wave energy was allowed to propagate along $y$ direction) with an aspect ratio $\mathscr{b}_{r}=x / y=2$, where $x$ and $y$ are the length and width of the model domain, respectively. Clearly, the fully developed asymptotic energy $\varepsilon_{*}$ (frequency $\nu_{*}$ ) is lower (higher) in the 2D run (gray dotted lines in Fig. 3) due to the narrow geometry of the fetch. The differences between these asymptotic values can be quantified by

$$
\mathscr{R}_{q}=\left|\left(q_{2}-q_{1}\right) / q_{1}\right|,
$$

where $q_{1}$ and $q_{2}$ are the asymptotic values from the 1D (broad fetch) and 2D (narrow fetch) cases. For $\mathscr{A}_{r}=2$, the DIA-based models $\left(\mathrm{ST}^{D}\right.$ and $\mathrm{ST} 4^{D}$ ) show $\mathscr{B}_{\varepsilon_{*}} \sim 10 \%$ and $\mathscr{B}_{\nu *}=2 \%$, whereas these metrics for ST $6^{G}$ and ST6 ${ }^{W}$ are markedly lower $\left(\mathscr{B}_{\varepsilon *} \sim 5 \%\right.$ and $\mathscr{B}_{\nu *} \leq 1 \%$ ) (Table 2). Results for $2 \mathrm{D}$ runs with higher aspect ratios $\left(\mathscr{b}_{r}=5,10\right.$; i.e., narrower fetches) present similar features (Table 2). The wave spectrum from the DIA is generally too broad (e.g., Hasselmann et al. 1985; Young et al. 1987) and thus becomes more constrained by the narrowness of the fetch, explaining the results we obtained here. An intuitive indication of these results is 

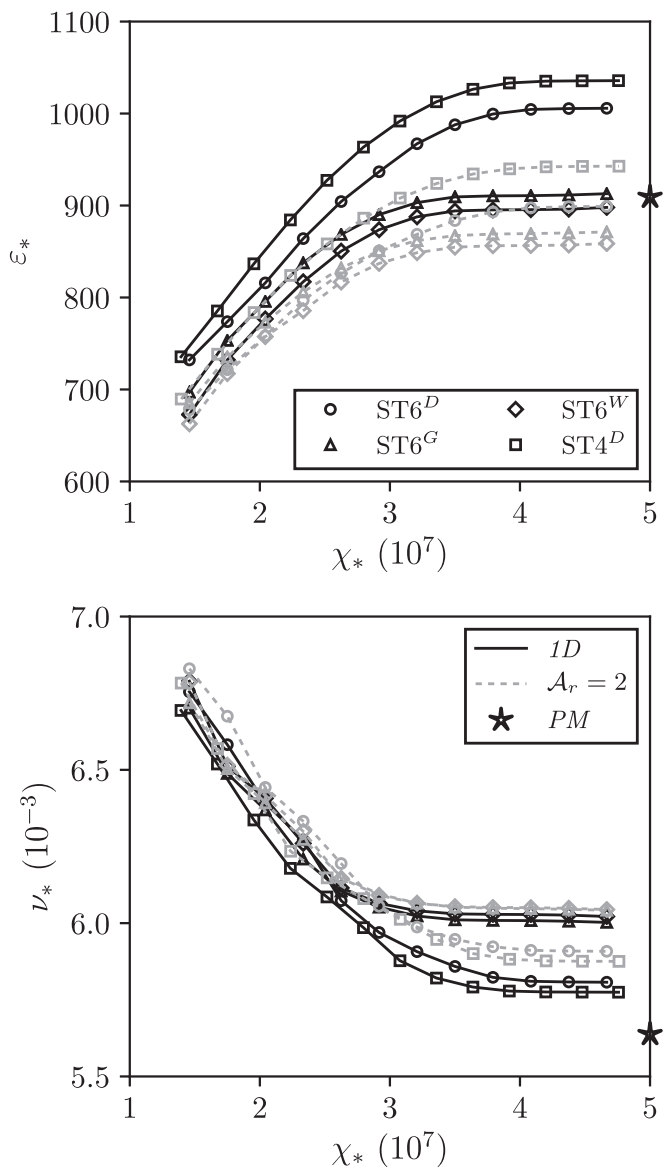

FIG. 3. Fetch-limited simulations from the third grid only $\left(N_{x}=40, \Delta x=250 \mathrm{~km} \rightarrow x=10000 \mathrm{~km}\right)$. The black solid lines with markers show the $1 \mathrm{D}$ (broad fetch) model results (i.e., the propagation of the wave energy along the $y$ direction is turned off), the gray dashed lines with markers represent 2D (narrow fetch) model results (i.e., wave energy is allowed to propagate along the $y$ direction) where the aspect ratio of the model domain is $\mathscr{b}_{r}=x / y=2$. The black asterisks highlight the PM asymptotic limits [(17)].

the DIA-based models may have problems simulating mature waves in narrow bays/lakes.

\section{Duration-limited wave growth}

The previous section focuses on integrated wave parameters (e.g., significant wave height $H_{s}$ and peak frequency $f_{p}$ ) only. However, as demonstrated by many previous studies (e.g., Banner and Young 1994; Alves and Banner 2003; Romero and Melville 2010b; Resio et al. 2016), high skills in predicting those bulk parameters do not necessarily guarantee high accuracies in modeled spectral shape. In this section, we will analyze simulated wave spectra in a much detailed way, mainly using spectral metrics suggested by RM10 and Resio et al. (2016). Most attention is dedicated to studying the following:

1) to what extent modeled spectra reflect measured properties of ocean waves, and

2) how well the GMD configuration presented in the previous section represents the exact solutions of $S_{\text {nl }}$ (i.e., WRT).

A single-grid-point, duration-limited wave growth experiment is selected here, mainly due to its computational efficiency and its reduced sensitivity to numerical errors (e.g., RBW12). The model setup is the same as the one used in fetch-limited simulations, except that the directional grid is refined from $\Delta \theta=10^{\circ}$ to $\Delta \theta=5^{\circ}{ }^{12}$ It is, however, particularly noteworthy that in the duration-limited simulations, the highfrequency spectral tail evolves freely without any prescribed slope.

\section{a. Equilibrium and saturation ranges}

Based on a dimensional analysis, Phillips (1958) proposed that the high-frequency range of the wave spectrum should follow a form

$$
E(f)=\alpha_{P} g^{2}(2 \pi)^{-4} f^{-5},
$$

if the wave breaking term $S_{\mathrm{ds}}$ is dominant in this specific frequency range, where $\alpha_{P}$ is the so-called Phillips constant. Toba (1973) argued the role of wind stress is also essential for small scale waves, and thus $E(f)$ should be alternatively parameterized as

$$
E(f)=\alpha_{T} u_{*} g(2 \pi)^{-3} f^{-4},
$$

where $\alpha_{T}$ is known as Toba's "constant." Later, assuming all the three physical processes $\left(S_{\mathrm{in}}, S_{\mathrm{ds}}, S_{\mathrm{nl}}\right)$ are important and comparable, Phillips (1985) reached the same form as (20). With the theoretical and observational progress in ocean waves over the past several decades (Hasselmann et al. 1973; Forristall 1981; Donelan et al. 1985; Ewans and Kibblewhite 1990; KC92; Hwang and Wang 2001; Resio et al. 2004; Babanin 2010; Lenain and Melville 2017; Zakharov 2018, among others), it has been gradually recognized that the equilibrium range $\left(\propto f^{-4}\right)$ and saturation range $\left(\propto f^{-5}\right)$ could coexist in wave spectra, with the equilibrium range typically located between $1.5 f_{p}$ and $3 \sim 3.5 f_{p}$ (Donelan et al. 1985; Resio et al. 2004) and the saturation range being applicable at higher frequencies.

\footnotetext{
${ }^{12}$ In the ST6 ${ }^{G}$ simulation, a localized nonlinear filter (Tolman 2011) was included to suppress spurious high-frequency noise in wave spectra.
} 
TABLE 2. Relative differences between the asymptotic values of the dimensionless energy and frequency $\left(\mathscr{B}_{\varepsilon_{*}}\right.$ and $\mathscr{R}_{\nu_{*}}$, respectively) from the 1D (broad fetch) model run and the 2D (narrow fetch) model runs with different aspect ratios (i.e., , $\left.b_{r}=x / y=2,5,10\right)$.

\begin{tabular}{|c|c|c|c|c|c|c|}
\hline & \multicolumn{2}{|c|}{$\mathscr{b}_{r}=2$} & \multicolumn{2}{|c|}{$\mathscr{A}_{r}=5$} & \multicolumn{2}{|c|}{$\mathscr{C}_{r}=10$} \\
\hline & $\mathscr{R}_{\varepsilon *}(\%)$ & $\mathscr{R}_{\nu *}(\%)$ & $\mathscr{B}_{\varepsilon *}(\%)$ & $\mathscr{B}_{\nu *}(\%)$ & $\mathscr{B}_{\varepsilon *}(\%)$ & $\mathscr{B}_{\nu *}(\%)$ \\
\hline $\mathrm{ST}^{D}$ & 11 & 2 & 22 & 4 & 32 & 8 \\
\hline $\mathrm{ST}^{G}$ & 5 & 1 & 10 & 2 & 18 & 5 \\
\hline ST $6^{W}$ & 4 & 0 & 10 & 1 & 16 & 2 \\
\hline $\mathrm{ST}^{D}{ }^{D}$ & 9 & 2 & 19 & 5 & 29 & 7 \\
\hline
\end{tabular}

Figure 4 shows the evolution in time of the modeled omnidirectional frequency spectrum $E(f)$ and saturation spectrum $B(f)$ over $48 \mathrm{~h}$ of duration-limited simulations, where

$$
B(f)=k^{3} F(k)=\frac{(2 \pi)^{4} f^{5} E(f)}{2 g^{2}} .
$$

Inspection of Fig. 4 suggests that all the three ST6 models (i.e., ST6 ${ }^{D}$, ST6 ${ }^{G}$, and ST6 ${ }^{W}$ in Figs. $4 \mathrm{a}, \mathrm{c}$ ) yield a clear transition from a power law of approximately $f^{-4}$ to the power law of about $f^{-5}$. At intermediate frequencies, wave spectra from ST4 ${ }^{D}$ (Fig. 4e) also follow the slope of $f^{-4}$ very well, whereas they fail to present the saturation range $E(f) \propto f^{-5}$ at higher frequencies. Consistent with ZBRY15 (their Fig. 5), here we find $\mathrm{ST} 4^{D}$ gives a high-frequency tail roughly proportional to $f^{-4.5}$. Another important result is that wave spectra from $\mathrm{ST}^{G}$ are in excellent agreement with those from ST6 ${ }^{W}$ (Fig. 4c), indicating the high accuracy of the GMD approach in reproducing exact solutions of $S_{\mathrm{nl}}$. As expected, $E(f)$ from ST6 ${ }^{W}$ and $\mathrm{ST}^{G}{ }^{G}$ are narrower in frequency space than those from DIA-based models (Fig. 4c vs Figs. 4a,e; see also our Figs. 7 and 8).

The saturation spectra $B(f)$ from ST $6^{D}$ (Fig. $4 \mathrm{~b}$ ), for different wave ages $c_{p} / u_{*}$ (where $c_{p}$ is the peak phase velocity), converge at frequencies between 0.3 and $0.6 \mathrm{~Hz}$ to a constant level (hereafter $B_{c}$ ), indicating that, for $U_{10}=20 \mathrm{~m} \mathrm{~s}^{-1}, B(f)$ at this frequency range is practically independent of the stage of development of ocean waves. It is worth mentioning that for lower winds (e.g., $U_{10}<15 \mathrm{~m} \mathrm{~s}^{-1}$ ), the dependence of $B(f)$ at saturation ranges on wave age $c_{p} / u_{*}$ or wind speed $U_{10}$ is clearly visible (Ewans and Kibblewhite 1990, their Fig. 3). RM10 found that the average of $B(f)$ over the interval of $k \in[0.75,2] \mathrm{rad} \mathrm{m}^{-1}$ (vertical dashed lines in Figs. $4 \mathrm{~b}, \mathrm{~d}, \mathrm{f})$ are $(8 \pm 2) \times 10^{-3}$ for $U_{10}>10 \mathrm{~m} \mathrm{~s}^{-1}$. The value of $B(f)$ given by $\mathrm{ST}^{D}$ over this wavenumber range is about $7 \times 10^{-3}$, falling within the measured range of RM10. It is also noteworthy that the $\mathrm{ST}^{D}$. favored constant level $B_{c}$ of $7 \times 10^{-3}$ is in excellent agreement with field measurements from Babanin and Soloviev (1998a; $\left.B_{c}=6.6 \times 10^{-3}\right)$ and Lenain and Melville (2017; $\left.B_{c}=7 \times 10^{-3}\right)$. ST6 ${ }^{G}$ (Fig. 4d) and ST6 ${ }^{W}$ show similar $B(f)$ except $B_{c}$ is slightly lower $(\sim 6 \times$ $\left.10^{-3}\right) .{ }^{13}$ For $f>0.6 \mathrm{~Hz}, B(f)$ from ST6 models starts to increase again, which may not be impossible as pointed out by Lenain and Melville (2017). As seen from Fig. $4 \mathrm{f}, \mathrm{ST} 4^{D}$ overestimates $B(f)$ at spectral tails as a result of the deviation of $E(f)$ from the $f^{-5}$ form.

To scrutinize the equilibrium range, we present Toba's parameter $\alpha_{T}$ in Fig. 5 as estimated from modeled spectra using

$$
\alpha_{T}=\frac{1}{f_{u}-f_{l}} \int_{f_{l}}^{f_{u}} \frac{(2 \pi)^{3} E(f) f^{4}}{u_{*} g} d f,
$$

where $f_{l}$ and $f_{u}$ are the lower and upper limits of integration. For consistency with RM10, we adopted $f_{l}=1.5 f_{p}$ and $f_{u}=0.29 \mathrm{~Hz} .{ }^{14}$ The values of $\alpha_{T}$ from all three ST6 models $\left(\mathrm{ST}^{D}{ }^{D}, \mathrm{ST}^{G}\right.$, and ST6 ${ }^{W}$ ) are quite close, and higher than those from RM10. Nonetheless, they are consistent with values suggested by Resio et al. (2004) for most wave ages and by Hwang et al. (2000a) for $c_{p} / u_{*} \sim 25$. For very young waves $\left(c_{p} / u_{*} \sim 10\right)$, ST6-computed $\alpha_{T}$ is noticeably lower because in such cases, equilibrium ranges are fairly narrow or might not exist (see our Fig. 6 and Babanin 2010). ST $4^{D}$ overpredicts $\alpha_{T}$ compared with ST6 models (ST6 ${ }^{D}$, $\mathrm{ST}^{G}{ }$, and $\mathrm{ST}^{W}{ }^{W}$ ) and the three just-mentioned field studies. But for $c_{p} / u_{*} \leq 20, \mathrm{ST} 4^{D}$-modeled $\alpha_{T}$ is still within the range of $[0.06,0.11]$ as summarized in Phillips (1985).

\section{b. Transition frequency}

Since ST6 models are able to produce a transition behavior from $E(f) \propto f^{-4}$ to $E(f) \propto f^{-5}$ (Fig. 4), it is interesting to check how well the transition frequency $f_{t}$ given by ST6 compares with previous studies.

\footnotetext{
${ }^{13}$ The $B(f)$ from $\mathrm{ST}^{W}$ is very close to that from $\mathrm{ST}^{G}$ (see Fig. 6), and thus not shown here for the clarity of this figure.

${ }^{14}$ The upper limit frequency $f_{u}=0.29 \mathrm{~Hz}$ corresponds to the highest resolved wavenumber $\left(0.35 \mathrm{rad} \mathrm{m}^{-1}\right)$ by the Airborne Topographic Mapper utilized in RM10.
} 

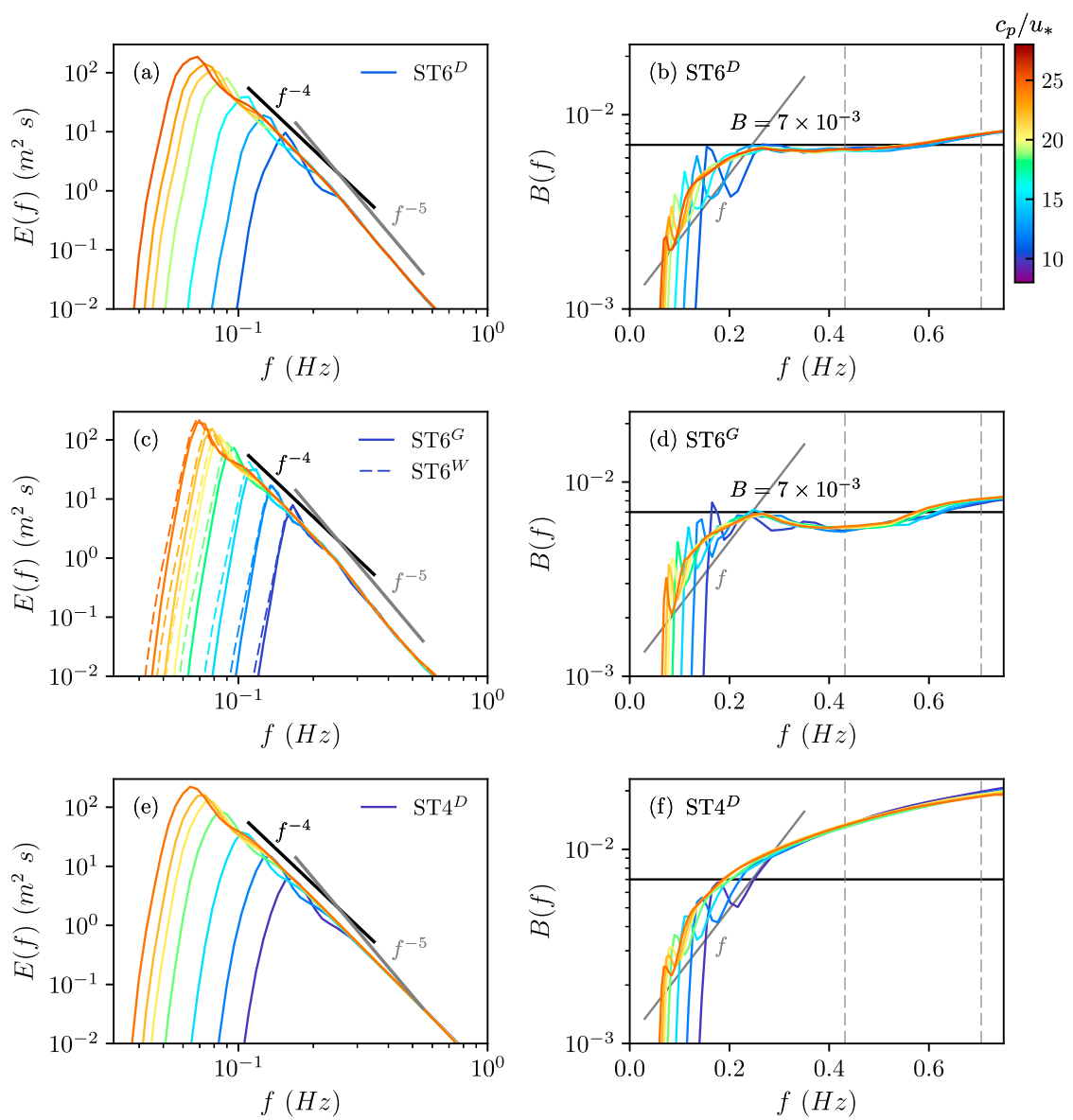

FIG. 4. The evolution of (left) omnidirectional frequency spectrum $E(f)$ and (right) saturation spectrum $B(f)$ from (a),(b) ST $6^{D}$, (c), (d) ST6 ${ }^{G}$ [and ST $6^{W}$; dashed lines in (c)], and (e),(f) ST $4^{D}$. Only spectra at $t=2,4,8,16,24,32,48 \mathrm{~h}$ are displayed and colorcoded according to the wave age $c_{p} / u_{*}$. In (a), (c), and (e), reference slopes proportional to $f^{-4}$ and $f^{-5}$ are shown as solid black and gray lines, respectively. The black lines in (b), (d), and (f) correspond to the converging constant saturation level $B=7 \times 10^{-3}$ of field measurements from Lenain and Melville (2017), and the gray line is a reference spectral slope proportional to $f$.

Forristall (1981) analyzed over 4000 wave spectra measured in situ and found that $f_{t}$ could be determined by

$$
f_{t} u_{*} / g=0.0275 \text {. }
$$

Based on an analysis of wave spectra collected from various wave-growth experiments, KC92 suggested that the transition from a $f^{-4}$ to $f^{-5}$ tail occurred at

$$
2 \pi f_{t} U_{10} / g \approx 5 .
$$

Similar to Forristall (1981), RM10 derived $f_{t}$ through finding the intersection of (19) and (20):

$$
2 \pi f_{t} u_{*} / g=\frac{\alpha_{P}}{\alpha_{T}}
$$

where the authors adopted $\alpha_{P}=2 B=0.016$ and $\alpha_{T}=$ $0.016\left(c_{p} / u_{*}\right)^{0.53}$ (blue line in Fig. 5). Using an alternative scaling for the equilibrium range as suggested by Resio et al. (2004),

$$
E(f)=\alpha_{R}\left[\left(U_{10}^{2} c_{p}\right)^{1 / 3}-u_{0}\right] g(2 \pi)^{-3} f^{-4} .
$$

Babanin (2010) quantified $f_{t}$ by

$$
2 \pi f_{t}\left[\left(U_{10}^{2} c_{p}\right)^{1 / 3}-u_{0}\right] / g=\frac{\alpha_{P}}{\alpha_{R}},
$$

where $\alpha_{R}=6.09 \times 10^{-3}$ and $u_{0}=3.25 \mathrm{~m} \mathrm{~s}^{-1}$ according to Resio et al. (2004) and $\alpha_{P}$ is a wave age-dependent spectrum-tail level as proposed by Babanin and Soloviev (1998a, gray solid line in our Fig. 7a). 


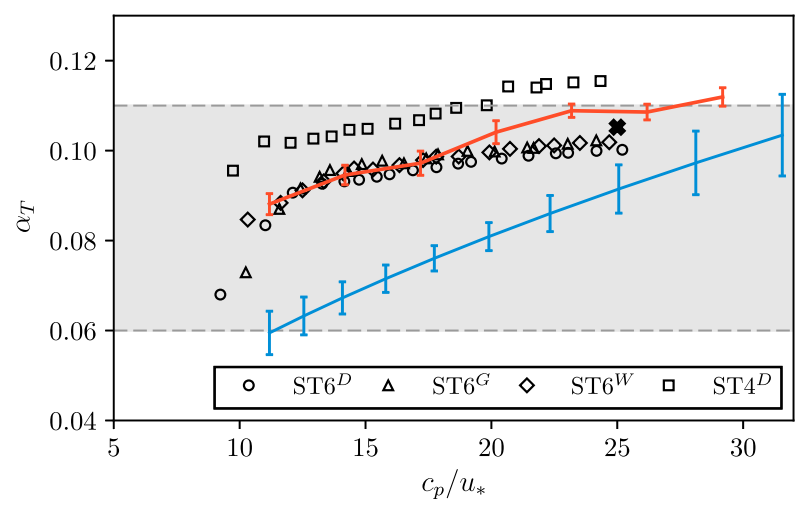

FIG. 5. Toba's parameter $\alpha_{T}$ vs the wave age $c_{p} / u_{*}$ as simulated by $\mathrm{ST}^{D}{ }^{\text {(circles), } \mathrm{ST}^{G}}$ (triangles), $\mathrm{ST}^{W}$ (diamonds), and ST4 ${ }^{D}$ (squares). The solid red and blue lines with error bars show measurements from Resio et al. (2004) and Romero and Melville (2010a). The thick $\times$ is the measurement from Hwang et al. (2000a), collected for a mature wind sea under quasi-steady winds $U_{10}=9.5 \mathrm{~m} \mathrm{~s}^{-1}$. The gray shaded region indicates the range of historical measurements summarized in Phillips (1985). Figure adapted from Romero and Melville (2010a).

In Fig. 6 we replotted $B(f)$ from ST6 models against the dimensionless frequency $f / f_{p}$, together with $f_{t}$ estimated from (23)-(27). As anticipated, $\mathrm{ST}^{G}$ and $\mathrm{ST}^{W}$ match the $f^{-4}$ slope better than $\mathrm{ST}^{D}$ for $1.5 f_{p}<f<2-3 f_{p}$, particularly when $c_{p} / u_{*}>15$. $\mathrm{ST}^{D}$ presents a slope close to $f^{-13 / 3}$ at such frequency regions for those wave ages ${ }^{15}$ (dashed line in Fig. 6a). Visual inspection of this figure suggests $f_{t}$ from the ST6 models is generally compatible with Forristall's estimation. The $f_{t}$ increases from below $2 f_{p}$ for very young waves to above $4 f_{p}$ for old wind seas; $f_{t}$ from KC92 is on average $0.5 f_{p}-f_{p}$ higher. As already reported by RM10, their $f_{t}$ is also higher than Forristall's values; but the difference gradually reduces as waves develop. Unlike the former three studies, Babanin (2010) favors an $f_{t}$ first increases with wave age and then decreases for $c_{p} / u_{*}>17$. This counterintuitive behavior is mainly attributed to the use of the wave age-dependent $\alpha_{P}$ (Babanin and Soloviev 1998a) in (27). During the 1990s, the form of the high-frequency wave spectrum $E(f) \propto f^{n}$ was in dispute (e.g., Young 1999, p. 123). Babanin and Soloviev (1998a) adopted the Joint North Sea Wave Project (JONSWAP) form (Hasselmann et al. 1973) for obtaining $\alpha_{P}$ from their field data. Unavoidably, their calculation of $\alpha_{P}$ must have included contributions

\footnotetext{
${ }^{15}$ The theoretical analysis in Resio et al. (2016) suggests the DIA parameterization for $S_{\mathrm{nl}}$ alone favors the spectral form $E(f) \sim f^{-10 / 3}$. The slope $f^{-13 / 3}$ we obtained, deviating from theirs, is probably due to the simultaneous consideration of all source terms (i.e., $\left.S_{\mathrm{in}}, S_{\mathrm{nl}}, S_{\mathrm{ds}}\right)$.
}
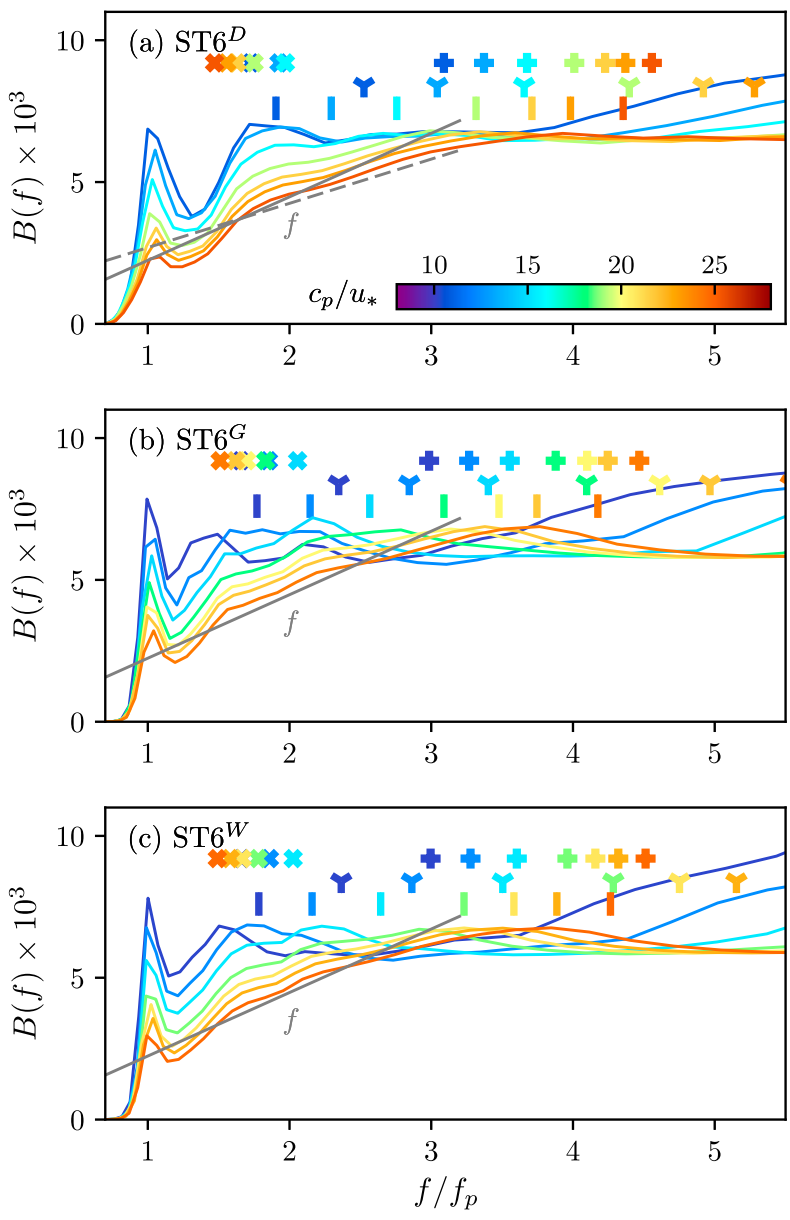

FIG. 6. As in Fig. 4, but for $B(f)$ against the dimensionless frequency $f / f_{p}$ according to (a) $\mathrm{ST}_{6}{ }^{D}$, (b) $\mathrm{ST}^{G}$, and (c) ST6 ${ }^{W}$. The color-coded markers "|", "Y", "+", and " $\times$ " highlight transition frequencies $f_{t}$ for corresponding wave ages as estimated by Forristall (1981), KC92, RM10, and Babanin (2010), respectively. Note that only horizontal coordinates of these markers are meaningful. The gray solid (dashed) line highlights the reference spectral slope proportional to $f\left(f^{2 / 3}\right)$.

from the equilibrium range, particularly for high wave ages. This is clearly demonstrated in our Fig. 7a: the modeled spectra with a nearly constant saturation level correspond to the JONSWAP spectra with an $\alpha_{P}$ value agreeing well with that from Babanin and Soloviev (1998a) (see the next subsection for more details).

\section{c. Spectral peakedness}

The spectral peakedness is a critical metric linked to the modulational instability of spectral waves and thus is frequently utilized in the freak wave literature (e.g., Janssen 2003; Onorato et al. 2006; Ribal et al. 2013). To examine modeled peakedness, we made an attempt to fit the generalized JONSWAP spectral form to each model 

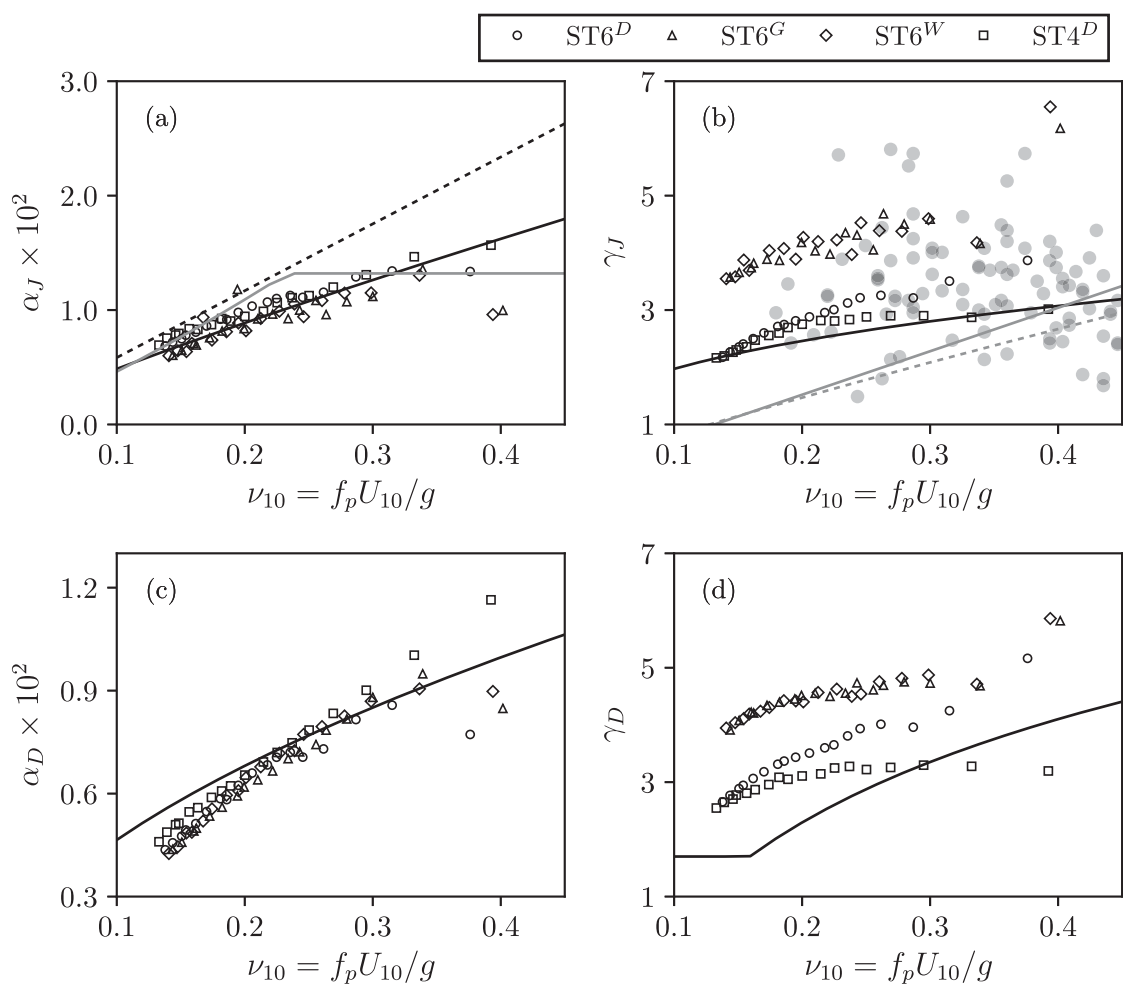

FIG. 7. The (left) high-frequency energy level $\alpha$ and (right) peak enhancement factor $\gamma$ vs the dimensionless frequency $\nu_{10}=f_{p} U_{10} / g$ for wave spectra of (a),(b) the JONSWAP form $\left(\propto f^{-5}\right)$ (Hasselmann et al. 1973) and (c),(d) the Donelan et al. (1985) form $\left(\propto f^{-4}\right)$. The relationships in (a) and (b) are Hasselmann et al. (1976; black solid lines), Janssen [2004; black dotted line for his (2.109)], Babanin and Soloviev (1998a; gray solid lines), and Lewis and Allos (1990; gray dotted line). The gray filled circles in (b) are the JONSWAP data sourced from Young (1999, his Fig. 5.17). The dependences in (c) and (d), shown as the solid lines, are defined in Donelan et al. (1985).

spectrum. Following Young (2006), the generalized JONSWAP expression reads

$$
\begin{aligned}
E(f)= & \alpha g^{2}(2 \pi)^{-4} f_{p}^{-(5+n)} f^{n} \exp \left[\frac{n}{4}\left(\frac{f}{f_{p}}\right)^{-4}\right] \\
& \times \gamma^{\exp \left[-\left(f-f_{p}\right)^{2} / 2 \sigma^{2} f_{p}^{2}\right]}
\end{aligned}
$$

where $\alpha$ is the high-frequency energy level, $\gamma$ is the peak enhancement factor, and $\sigma$ is the peak width parameter. For $n=-5$, (28) corresponds to the JONSWAP form established by Hasselmann et al. (1973) and for $n=-4$ to the form proposed by Donelan et al. (1985).

Both of these forms of (28) with $n=-5$ and $n=-4$ were applied in the curve fitting process, and spectral parameters $\alpha$ and $\gamma$ obtained from each of the best-fit spectra are illustrated in Fig. 7. The fit was attempted over the full-frequency range (i.e., $f_{i} \in[0.037,1.027] \mathrm{Hz}$ ) with a nonlinear least squares method,${ }^{16}$ which minimizes

\footnotetext{
${ }^{16}$ The least_squares function from the scipy.optimize package.
}

the cost function $\mathscr{E}=\sum_{i}\left[E^{\prime}\left(f_{i}\right)-E\left(f_{i}\right)\right]^{2}$, where $E^{\prime}\left(f_{i}\right)$ and $E\left(f_{i}\right)$ denote the fitted and simulated discrete wave spectra, respectively (see also Battjes et al. 1987). For the JONSWAP form (Figs. 7a,b), the DIA-based models $\left(\mathrm{ST}^{D}{ }^{D}\right.$ and ST $4^{D}$ ) yield an $\alpha$ marginally higher than that from $\mathrm{ST}_{6}{ }^{G}$ and $\mathrm{ST} 6^{W} ; \alpha$ from all these four models is generally consistent with the power law proposed by Hasselmann et al. (1976) and slightly lower than power laws described in Babanin and Soloviev (1998a) and Janssen (2004). The most striking result in Fig. $7 \mathrm{~b}$ is that $\gamma$ from ST6 ${ }^{G}$ and ST6 ${ }^{W}$ is remarkably greater than that predicted by Hasselmann et al. (1976), Lewis and Allos (1990), and Babanin and Soloviev (1998a), although it is still within the range of values observed in JONSWAP experiment (Hasselmann et al. 1973; Young 1999); whereas $\mathrm{ST}^{D}$ and ST4 ${ }^{D}$ conform to the power law of Hasselmann et al. (1976) much better. For the form of Donelan et al. (1985) (Figs. 7c,d), $\alpha$ from the four models is fairly consistent and follows the dependence on wave age found by Donelan et al. (1985) reasonably well. In contrast, all 
models display a $\gamma$ generally higher than that from Donelan et al. (1985). In spite of these discrepancies, the consistent features of Fig. 7 are as follows: 1) $\gamma$ from $\mathrm{ST}_{4}{ }^{D}$ is in general the lowest, then $\mathrm{ST}^{D}{ }_{-}$ simulated $\gamma$ is marginally higher, and $\gamma$ from ST6 ${ }^{G}$ and ST ${ }^{W}$ is the highest; and 2) except for very young waves $\left(\nu_{10} \sim 0.4\right)$, both modeled $\alpha$ and $\gamma$ decrease as the wave develops $\left(\nu_{10} \downarrow\right)$, which is analogous to previous field studies (e.g., Hasselmann et al. 1976; Donelan et al. 1985; Babanin and Soloviev 1998a; Janssen 2004).

Another three metrics connected to spectral peakedness are illustrated in Fig. 8, including the spectral width $F_{w}[\nu$ in Babanin and Soloviev (1998a)], spectral narrowness $F_{n}\left[Q_{c}\right.$ in Rogers and van Vledder (2013)], and the Benjamin-Feir index (BFI; Janssen 2003; Onorato et al. 2006; Xiao et al. 2013):

$$
\begin{aligned}
F_{w} & =\frac{m_{0}}{f_{p} E\left(f_{p}\right)}, \\
F_{n} & =\frac{\max \left[E(f) / m_{0}\right]}{m_{-1} / m_{0}}, \text { and } \\
\mathrm{BFI} & =\frac{\sqrt{2} \delta}{2 \Delta f / f_{p}}=\frac{k_{p} H_{s}}{2 \sqrt{2} \Delta f / f_{p}},
\end{aligned}
$$

where $m_{n}$ is the $n$ th-order moment (appendix A) and $\Delta f$ is the half-width at the half-maximum of $E(f)$. Remarkably, similar to what we have seen in Fig. 7, the spectral narrowness $F_{n}$ (Fig. 8b) from ST6 ${ }^{G}$ and $\mathrm{ST}^{W}$ are clearly higher than that for DIA-based models $\left(\mathrm{ST}^{D}{ }^{D}\right.$ and $\left.\mathrm{ST} 4^{D}\right)$, and all the models underestimate spectral width $F_{w}$ from Krivinskii (1991) for most wave ages $\left(\nu_{10}<0.3\right.$; Fig. 8a). A close inspection of Fig. $8 \mathrm{c}$ suggests that $\mathrm{ST}^{W}{ }^{W}$ - and $\mathrm{ST}^{G}{ }^{G}$-simulated BFI appears unrealistically high (BFI $>1)$.

Therefore, a consistent finding from our Figs. 7 and 8 is that the WRT- and GMD-based models (i.e., ST6 ${ }^{W}$ and $\mathrm{ST}^{G}{ }^{G}$ ), contrary to our expectation, noticeably overestimate the peakedness/narrowness (or alternatively, underestimate the width) of wave spectra. Being an approximation to the WRT, the DIA provides a slightly improved, but still problematic in general, estimation of the spectral peakedness due to its inherent tendency to unrealistically broaden the exact solutions in frequency space. Although counterintuitive, this finding is remarkably supported by a recent numerical study by Annenkov and Shrira (2018). The authors showed (their Figs. 6 and 11) that relative to the WRT results based on the Hasselmann kinetic equation (Hasselmann 1962), their direct numerical
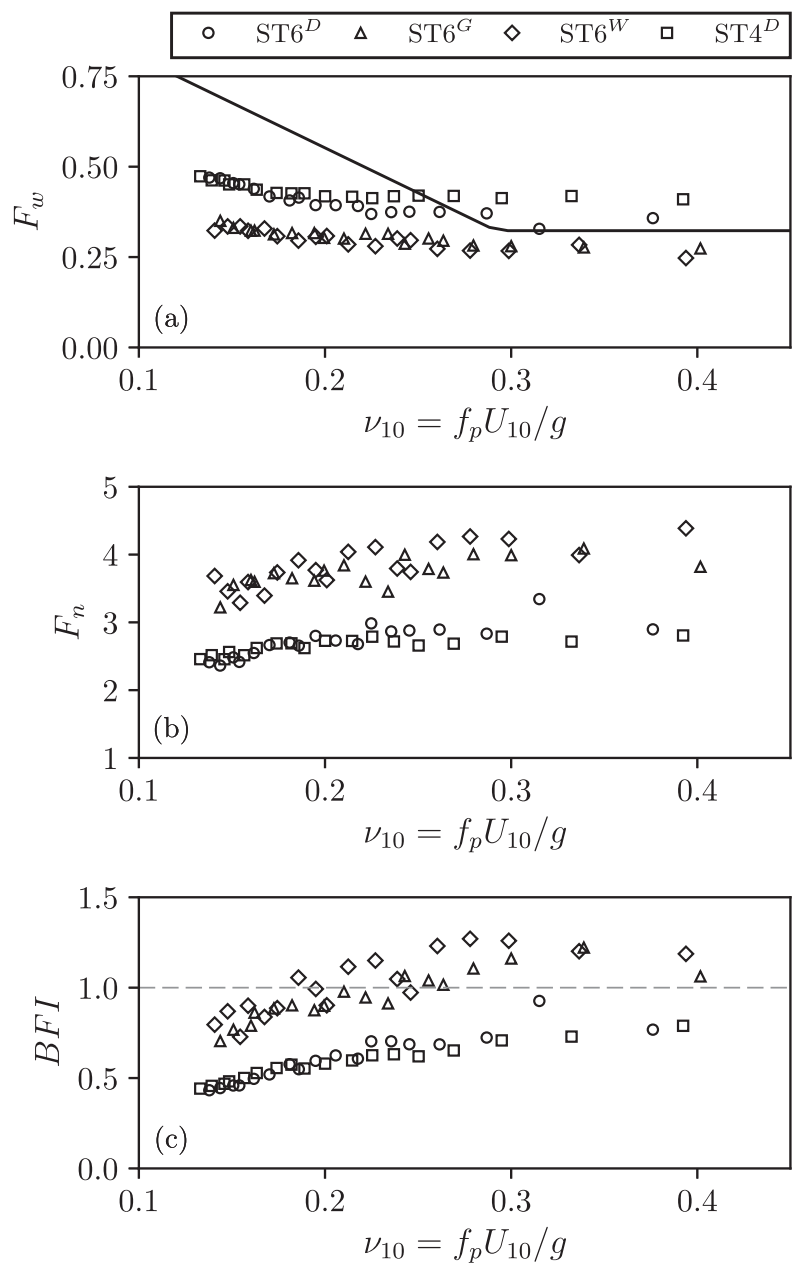

FIG. 8. The evolution of (a) spectral width $F_{w}$ (Babanin and Soloviev 1998a), (b) spectral narrowness $F_{n}$ (Rogers and van Vledder 2013), and (c) BFI (Onorato et al. 2006) against dimensionless frequency $\nu_{10}=f_{p} U_{10} / g$. The solid black line highlights the dependence of $F_{w}$ on wave development suggested by Krivinskii (1991).

simulations based on the Zakharov integrodifferential equation (Zakharov 1968) predict "considerably wider frequency spectra with much less pronounced peaks." Considering that the Zakharov equation is "the primitive equation for a weakly nonlinear wave field" and "does not employ any statistical assumptions," and considering that the Hasselmann kinetic equation can be derived from the Zakharov equation "by applying standard closure hypothesis," Annenkov and Shrira (2018) argued these systematic mismatches "call for revision of the fundamentals" of the Hasselmann kinetic equation. Besides, the poor performance of wave models in simulating the spectral peak implies a difficulty in predicting the occurrence of freak waves. 

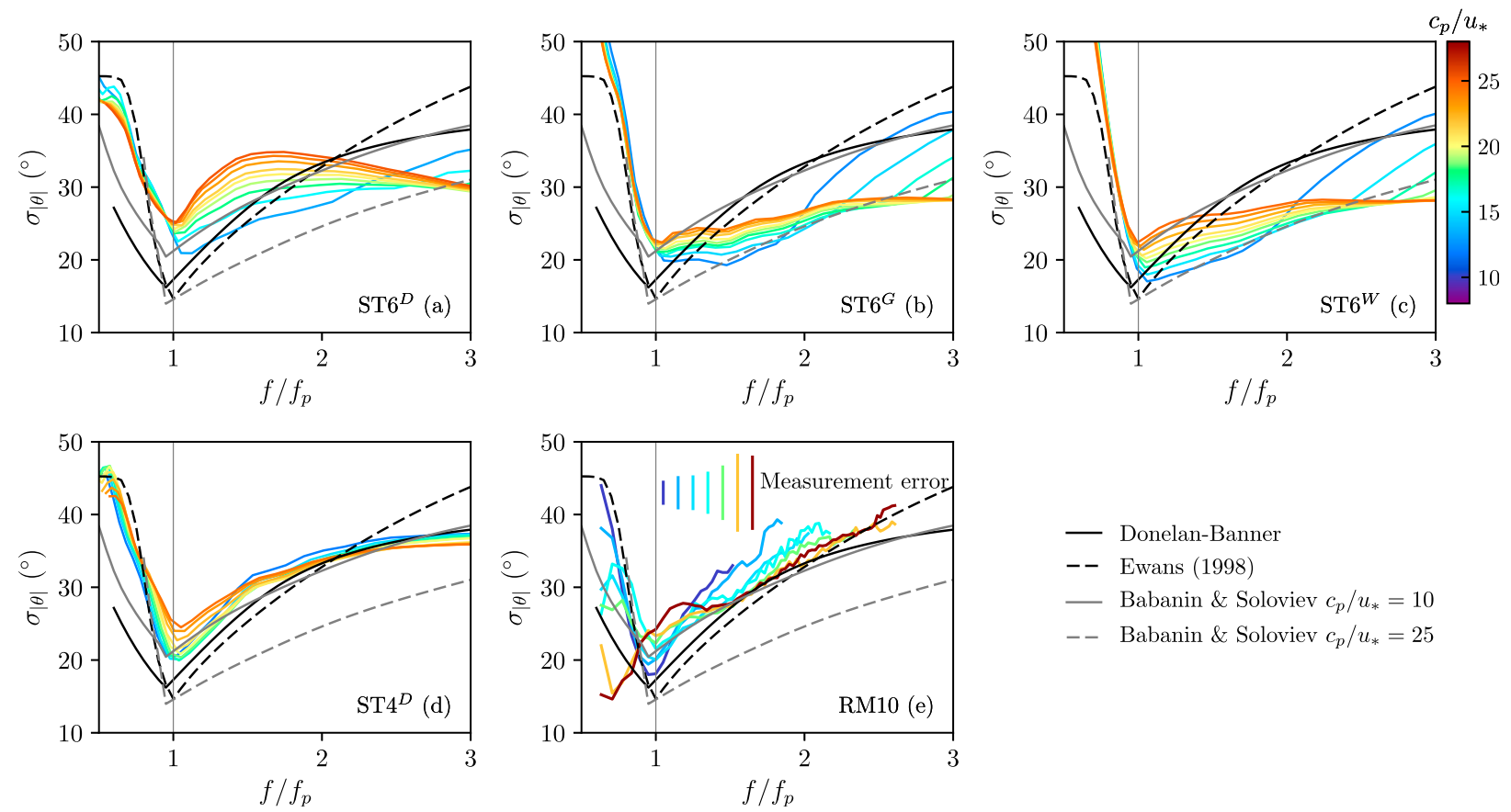

FIG. 9. Directional spreading $\sigma_{|\theta|}$ according to (a) $\mathrm{ST}^{D}$, (b) ST6 ${ }^{G}$, (c) $\mathrm{ST} 6^{W}$, (d) ST4 ${ }^{D}$, and (e) spatial measurements from RM10. The solid and dashed black lines illustrate $\sigma_{|\theta|}$ calculated from the directional distribution models of Donelan-Banner and Ewans (1998), respectively. The solid and dashed gray lines show $\sigma_{|\theta|}$ based on the form of Babanin and Soloviev (1998b) for $c_{p} / u_{*}=10$ and 25 , respectively. Also shown in (e) are measurement errors of peak spreading (i.e., $\sigma_{|\theta|}$ at $f_{p}$ ) from RM10 (vertical colored lines), starting from $3^{\circ}$ for the lowest wave age group $\left(c_{p} / u_{*} \sim 10\right)$ to $10^{\circ}$ for the largest wave age group $\left(c_{p} / u_{*} \sim 28\right)$.

\section{d. Directional properties}

The last two metrics selected in this section are associated with directional properties of wave spectra. The first metric is the directional spreading. For comparison purpose, here we adopt the definition described in RM10:

$$
\sigma_{|\theta|}=\frac{\int_{-\pi / 2}^{\pi / 2} F(f, \vartheta)|\vartheta| d \vartheta}{\int_{-\pi / 2}^{\pi / 2} F(f, \vartheta) d \vartheta},
$$

where $\vartheta$ is the angle relative to the dominant wave direction $(\vartheta \in[-\pi, \pi]$; see, e.g., Fig. 16 of RM10).

Figure 9 illustrates $\sigma_{|\theta|}$ from different models as a function of $f / f_{p}$. Three directional distribution parameterizations are also shown as references, including 1) the unimodal $\operatorname{sech}^{2}(\beta \theta)$ form proposed by Donelan et al. (1985) with the extension at high frequencies suggested by Banner (1990) (hereafter the Donelan-Banner form), where the parameter $\beta$ determines the spectral spread; 2) the bimodal form as described in Ewans (1998); and 3) the same $\operatorname{sech}^{2}(\beta \theta)$ form of Donelan et al. (1985) but with $\beta$ suggested in Babanin and Soloviev (1998b) (hereafter the
Babanin-Soloviev form). Note that unlike the former two forms, the latter form depends on the stage of wave development. Nonetheless, all these forms correspond to a $\sigma_{|\theta|}$ that is a minimum in the neighborhood of the spectral peak and then increasing toward lower and higher frequencies. Among the four wave models, ST4 ${ }^{D}$ (Fig. 9d) compares the best with the two wave age-independent parametric forms (black lines in Fig. 9). The directional spreading estimated by $\mathrm{ST}_{4}{ }^{D}$ agrees well with Ewans' values below the peak, and then starts to match DonelanBanner's prediction for higher frequencies. At $f_{p}$, $\mathrm{ST} 4^{D}$-computed wave spectra are moderately broader than measurements from Donelan et al. (1985) and Ewans (1998), but are still comparable to the spatial measurements from RM10 (Fig. 9e). On the other hand, wave spectra from ST $6^{D}$ (Fig. 9a) are too broad at $f_{p}-1.5 f_{p}$ and too narrow beyond $2 f_{p}$. The overestimation of spreading near the spectral peak by $\mathrm{ST}^{D}{ }^{D}$ is partially reduced by ST $6^{G}$ and ST6 ${ }^{W}$ (Figs. 9b,c) due to their more accurate computations of $S_{\mathrm{nl}}$. However, for $f>1.5 f_{p}, \sigma_{|\theta|}$ from ST6 ${ }^{G}$ and ST $6^{W}$ is fairly low, particularly when compared against measurements from Donelan et al. (1985), Ewans (1998), and RM10. The dependence of the peak spreading on $c_{p} / u_{*}$ deserves special attention. Wave models and observations 
from RM10 show an upward trend in $\sigma_{|\theta|}$ at $f \sim f_{p}$ as waves develop. This is, however, in contradiction to measurements from Babanin and Soloviev (1998b) and Donelan (2017), which suggest the peak of wave spectrum becomes narrower as $c_{p} / u_{*}$ increases (e.g., see gray lines in Fig. 9). It should be remarked that there is relatively large uncertainty associated with the peak spreading measurements $\left(\sigma_{|\theta|}\right.$ at $\left.f_{p}\right)$ by RM10. Their measurement error of peak spreading generally increases with wave age, and for $c_{p} / u_{*} \sim 28$, the error can be as large as $10^{\circ}$ (Fig. 9e). Similarly, the directional spreading estimated from $F(f, \theta)$ measured by in situ wave buoys is rather sensitive to instrument or analysis noise, in particular for the peak spreading, which is generally low (Kuik et al. 1988, their Fig. 3).

The second directional metric to be evaluated here is the bimodality of wave components above the spectral peak, as reported by a number of previous studies (Young et al. 1995; Ewans 1998; Hwang et al. 2000b; Lenain and Melville 2017; RM10, among others). Following Wang and Hwang (2001), for a given $f>f_{p}$ the angular separation between the two local maxima of $F(f, \theta)$ is quantified by the metric $\theta_{\text {lobe }}$ :

$$
\theta_{\text {lobe }}(f)=\frac{\left|\theta_{1}(f)\right|+\left|\theta_{2}(f)\right|}{2},
$$

where $\theta_{1}$ and $\theta_{2}$ are the locations of the maximum $F(f, \theta)$ on each side of the mean wave direction. With directional wave spectra measured with a heave-pitch-roll buoy, Ewans (1998) found that $\theta_{\text {lobe }}$ weakly depends on wave age, and its average over all stages of wave development ${ }^{17}$ can be fitted by

$$
\theta_{\text {lobe }}=0.5 \exp \left(5.453-2.750 f_{p} / f\right)
$$

for $f \geq f_{p}$. Similarly, RM10 also found a weak dependence of $\theta_{\text {lobe }}$ on wave age from their spatial measurements, showing that for $1.5 \leq\left(f / f_{p}\right)^{2} \leq 6.5$

$$
\begin{aligned}
\theta_{\text {lobe }}^{\prime} & =\theta_{\text {lobe }} \sqrt{c_{p} / u_{*} / 5} \\
& =-0.736\left(f / f_{p}\right)^{4}+12.9\left(f / f_{p}\right)^{2}-8.35 .
\end{aligned}
$$

Figure 10 presents $\theta_{\text {lobe }}^{\prime}$ estimated from the four wave models, together with measurements from Ewans (1998) and RM10. For low wave ages, ST6 $^{D}$ (Fig. 10a) is able to

\footnotetext{
${ }^{17}$ The measurements from Ewans (1998) had $U_{10}$ in between 4.6 and $18.3 \mathrm{~m} \mathrm{~s}^{-1}$, and inverse wave ages ranging from 0.7 to 1.4 . Taking the average $U_{10}$ as $12 \mathrm{~m} \mathrm{~s}^{-1}$, the mean wave age $c_{p} / u_{*}$ of his measurements corresponds to approximately 25 .
}

exhibit a bimodal structure, although $\theta_{\text {lobe }}^{\prime}$ is around $7^{\circ}$ lower than measurements. For high wave ages, however, wave spectra from $\mathrm{ST}^{D}$ generally lose the bimodality, as represented by $\theta_{\text {lobe }}^{\prime}=0^{\circ}$. ST $6^{G}$ and ST6 $^{W}$ (Figs. 10b,c) show enhanced ability to reproduce the bimodal behavior, except for fairly old waves $\left(c_{p} / u_{*} \sim 25\right)$. ST ${ }^{D}$ (Fig. 10d) exhibits a superior performance over $\mathrm{ST}^{D}$ in terms of its capacity to reproduce a bimodal spectral structure. Nonetheless, it appears that the dependence of $\theta_{\text {lobe }}^{\prime}$ from ST4 ${ }^{D}$ on wave development is relatively stronger than those from $\mathrm{ST}^{G}{ }$, ST6 ${ }^{W}$, and field measurements (Ewans 1998; RM10). The lobe ratios, defined by $r_{\text {lobe }}=\left[F\left(f, \theta_{1}\right)+F\left(f, \theta_{2}\right)\right] / 2 F(f, 0)$, from the four wave models are lower than values from RM10 for most of wave ages and thus are not reproduced here (section 5 of the SOM).

\section{Global hindcast}

After a thorough analysis of model skills in specifying the wave spectrum in academic tests, it is necessary to verify performances of $\mathrm{ST}^{D}$ and $\mathrm{ST}^{G}$ in realistic global hindcasts. ST6 ${ }^{W}$ is excluded from the analysis here because of its computational infeasibility at the global scale.

SABZ16 conducted a comprehensive study on the comparison and assessment of different source term packages (Tolman and Chalikov 1996; Janssen 2004; Ardhuin et al. 2010; ZBRY15) available in WW3 with a global hindcast of the year 2011. For easy intercomparison with other packages evaluated in SABZ16, we selected the same year (2011) in our simulations. The global model domain is bounded within $78^{\circ} \mathrm{S}-78^{\circ} \mathrm{N}$, with a resolution of $1 / 2^{\circ} \times 1 / 2^{\circ}$. The resolution of the spectral grid is $\Delta f / f=0.1$ and $\Delta \theta=10^{\circ}$ with frequencies ranging from 0.037 to $0.953 \mathrm{~Hz}$. As for SABZ16, we used winds from CFSv2 (Saha et al. 2014) as the external forcing $\left(\sim 0.2^{\circ}\right.$; 3 hourly), and $\beta_{\max }=1.25$ for ST4 ${ }^{D}$. It was shown that the CFSv2 winds of 2011 agreed well with altimeter $U_{10}$ observations (SABZ16, their Fig. 2), with $b_{n} \sim 2 \%$ and $\rho=0.93$. (The corresponding spatial distribution of the errors of CFSv2 winds is presented in Fig. B1 in appendix B.)

\section{a. Comparison against altimeters}

Figure 11 presents comparisons of the simulated wave height $H_{s}$ by different wave models and altimeter measurements from Young et al. (2017). Records of $H_{s}$ from four altimeters, including Jason-1/2, Envisat, and CryoSat-2, were sourced, leading to a large set of model-altimeter collocations $\left[\odot\left(10^{6}\right)\right]$. Relative to altimeter observations, the overall bias $b$ and RMSE 

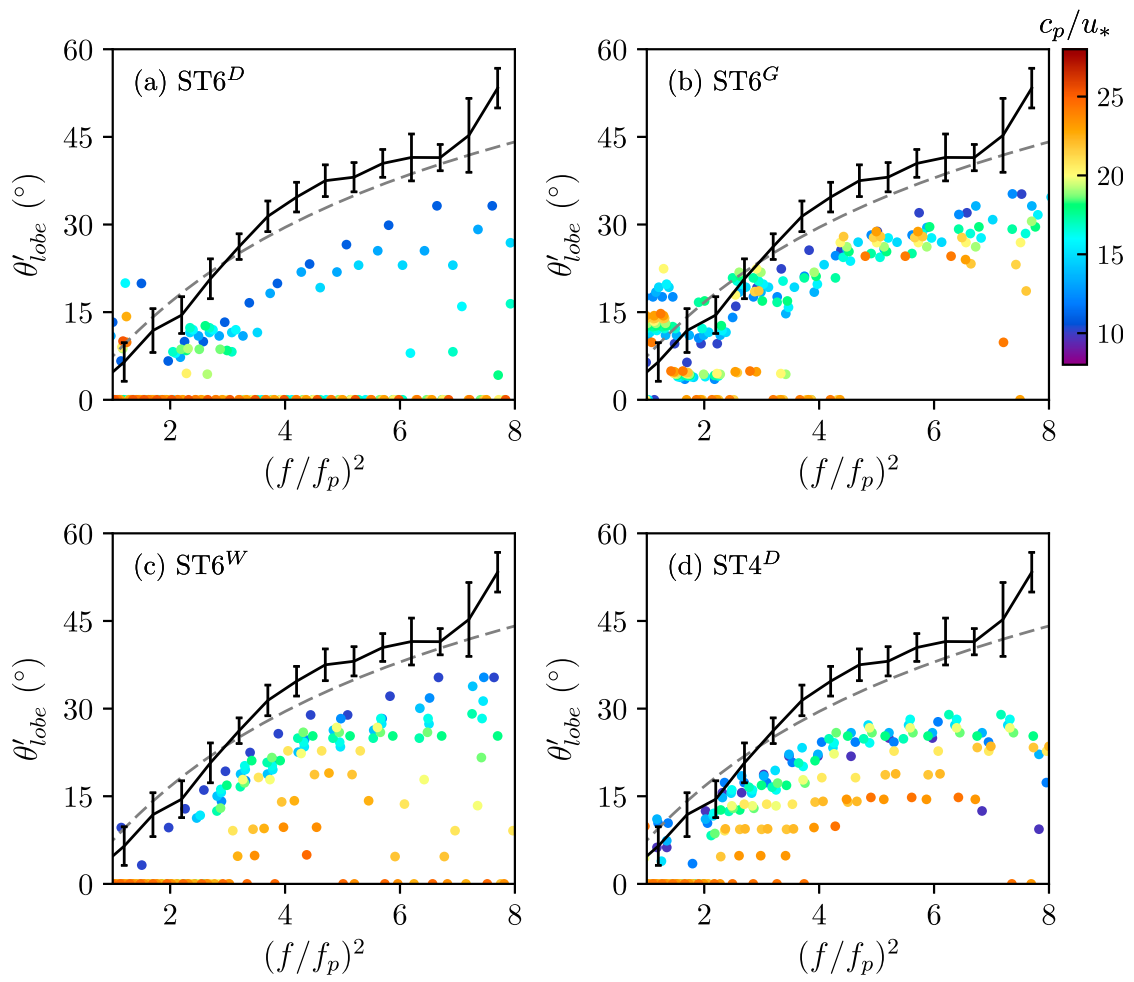

FIG. 10. The $\theta_{\text {lobe }}$, scaled with $\sqrt{c_{p} / u_{*} / 5}$, against $\left(f / f_{p}\right)^{2}$ according to (a) $\mathrm{ST}^{D}$, (b) $\mathrm{ST}^{G}$, (c) $\mathrm{ST}_{6}{ }^{W}$, and (d) $\mathrm{ST}_{4}{ }^{D}$. The black line with error bars show measurements collected by RM10, from which (35) was fit. The dashed line is $\theta_{\text {lobe }}^{\prime}$ estimated from Ewans' formula (34) with the mean wave age $c_{p} / u_{*}=25$ of his measurements.

$\epsilon$ in $\mathrm{ST}^{D}{ }^{D}$-simulated $H_{s}$ (Fig. 11a) are $0.04 \mathrm{~m}$ and $0.39 \mathrm{~m}$, respectively. The scatter index (SI) is low (0.14) and the correlation coefficient $\rho$ is as high as 0.96. The performance of $\mathrm{ST}^{G}{ }^{G}$ (Fig. 11b) is slightly improved, showing a $b$ of $0 \mathrm{~m}$, reduced $\epsilon(0.35 \mathrm{~m})$ and SI (0.13), and an elevated $\rho$ of 0.97 . ST4 ${ }^{D}$ (Fig. 11c) provides a remarkably similar skill to that of $\mathrm{ST}_{6}{ }^{G}$. Note that error metrics shown here are in good agreement with those from ZBRY15 (their Fig. 13), where the authors also found that $\mathrm{ST} 4^{D}$ was marginally superior to $\mathrm{ST} 6^{D}$ in modeling $H_{s}$ for the global basin.

The spatial distributions of the normalized bias $b_{n}$ and RMSE $\epsilon_{n}$ of simulated $H_{s}$ by each of the three models are illustrated in Fig. 12. Consistent with previous studies (e.g., Ardhuin et al. 2010; ZBRY15), $H_{s}$ values from all the three models (Figs. 12a,c,e) are biased low in the western Pacific Ocean, the majority of the Atlantic Ocean, and the tropical Indian Ocean, with a $b_{n}$ about $-5 \%$. The underestimation of $H_{s}$ becomes slightly more noticeable in the western tropical Pacific Ocean and the tropical Atlantic Ocean, particularly in the proximity of the Indonesia archipelago $\left(b_{n}<-10 \%\right)$. This could result from the bias in CFSv2 winds in equatorial zones (SABZ16; see our
Fig. B1), the neglect of shoreline reflection (Ardhuin et al. 2010), and the overblocking effect of obstruction grids utilized to represent unresolved islands (Tolman 2003). In contrast, the eastern Pacific Ocean and the Southern Ocean are dominated by positive biases in $H_{s}$. In the Southern Ocean, $b_{n}$ is roughly zonally distributed and increases toward the Antarctica from 5\% to $25 \%$. Ardhuin et al. (2011) demonstrated that including the blocking effect of small-size icebergs could reduce positive biases of $H_{s}$ south of $50^{\circ} \mathrm{S}$. All the three wave models also share a similar pattern of the normalized RMSE (Figs. 12b,d,f): $\epsilon_{n}$ in most of oceans is below $15 \%$, whereas regions such as the equatorial western Pacific Ocean and the midlatitudes in the North Atlantic Ocean correspond to a moderately higher $\epsilon_{n}(\sim 20 \%) ; \epsilon_{n}$ adjacent to Antarctica is the highest, and could be above $25 \%$. Once again, this is comparable to results from Ardhuin et al. (2010) and ZBRY15. A close inspection of Figs. 12b, 12d, and $12 \mathrm{f}$ suggests that $\mathrm{ST}^{G}{ }^{G}$ and ST $4^{D}$ perform slightly better than $\mathrm{ST} 6^{D}$, analogous to what we have seen in Fig. 11.

The geophysical mismatches presented in Fig. 12 can be partially, but not fully, explained by the errors of wind forcing (Fig. B1). For example, the overestimation 

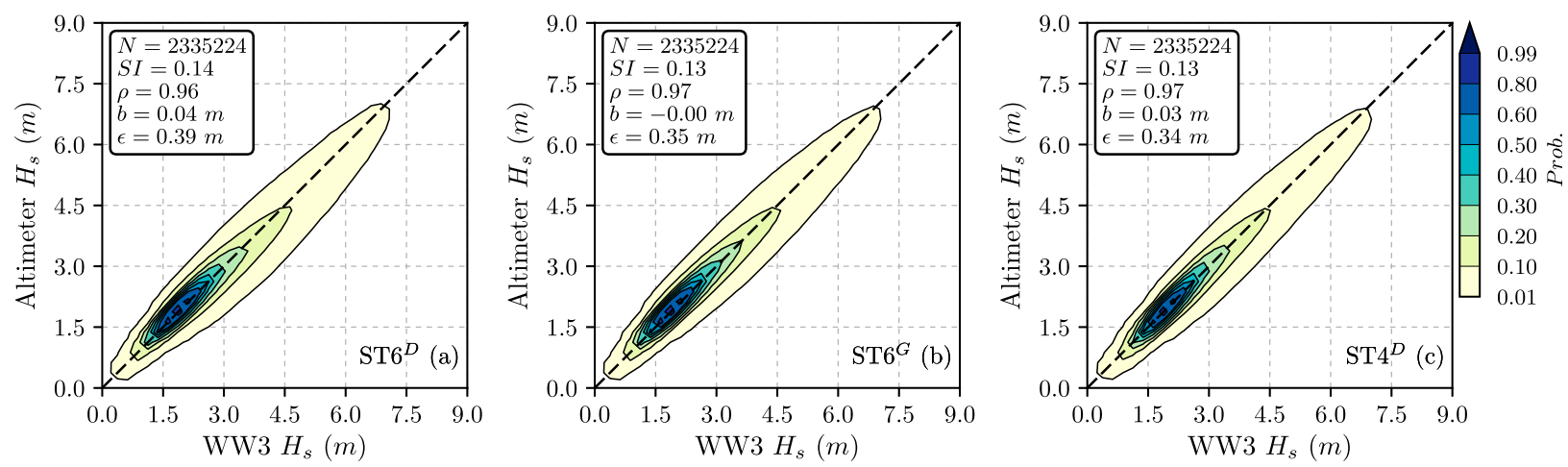

FIG. 11. Comparison of $H_{s}$ for the 2011 global hindcast between altimeters and wave model (a) ST6 ${ }^{D}$, (b) ST6 ${ }^{G}$, and (c) ST4 ${ }^{D}$. The dashed line is the 1:1 line. Error metrics printed in the inset of each panel includes the total number of model-altimeter collocations $N$, bias $b$, RMSE $\epsilon$, correlation coefficient $\rho$, and scatter index SI (see appendix A).

of $H_{s}$ in the eastern Bay of Bengal, the western Arabian Sea, the Hudson Bay, and the Argentine Sea can be attributed to the biased-high CFSv2 wind forcing. The overall negative bias of $H_{s}$ dominated in the central Pacific, Atlantic, and Indian Oceans is in line with the overall underestimation of $U_{10}$ by CFSv2 as well. Besides, the relatively high $\epsilon_{n}$ of the simulated $H_{s}$ present in the western midlatitude Pacific and Atlantic Oceans and the northern Indian Ocean correlates well with the comparatively high $\epsilon_{n}$ of $U_{10}$. Nonetheless, the signs of the biases of $H_{s}$ and $U_{10}$ in the eastern Pacific Ocean and the entire Southern
Ocean are opposite, suggesting that other physical factors are operative. A close inspection of Fig. 12 indicates that wave energy was overestimated in the zone of the southern westerlies and then these overestimated wave energy propagates northeastward as swell to the eastern coast of the South Pacific Ocean. A detailed analysis of the wave model biases in the Southern Ocean, as done by Ardhuin et al. (2011) and Rapizo et al. (2018), is left for future studies. The reader is also referred to Ardhuin et al. (2010, p. 1930) for further discussions about the spatial distribution of wave model errors.

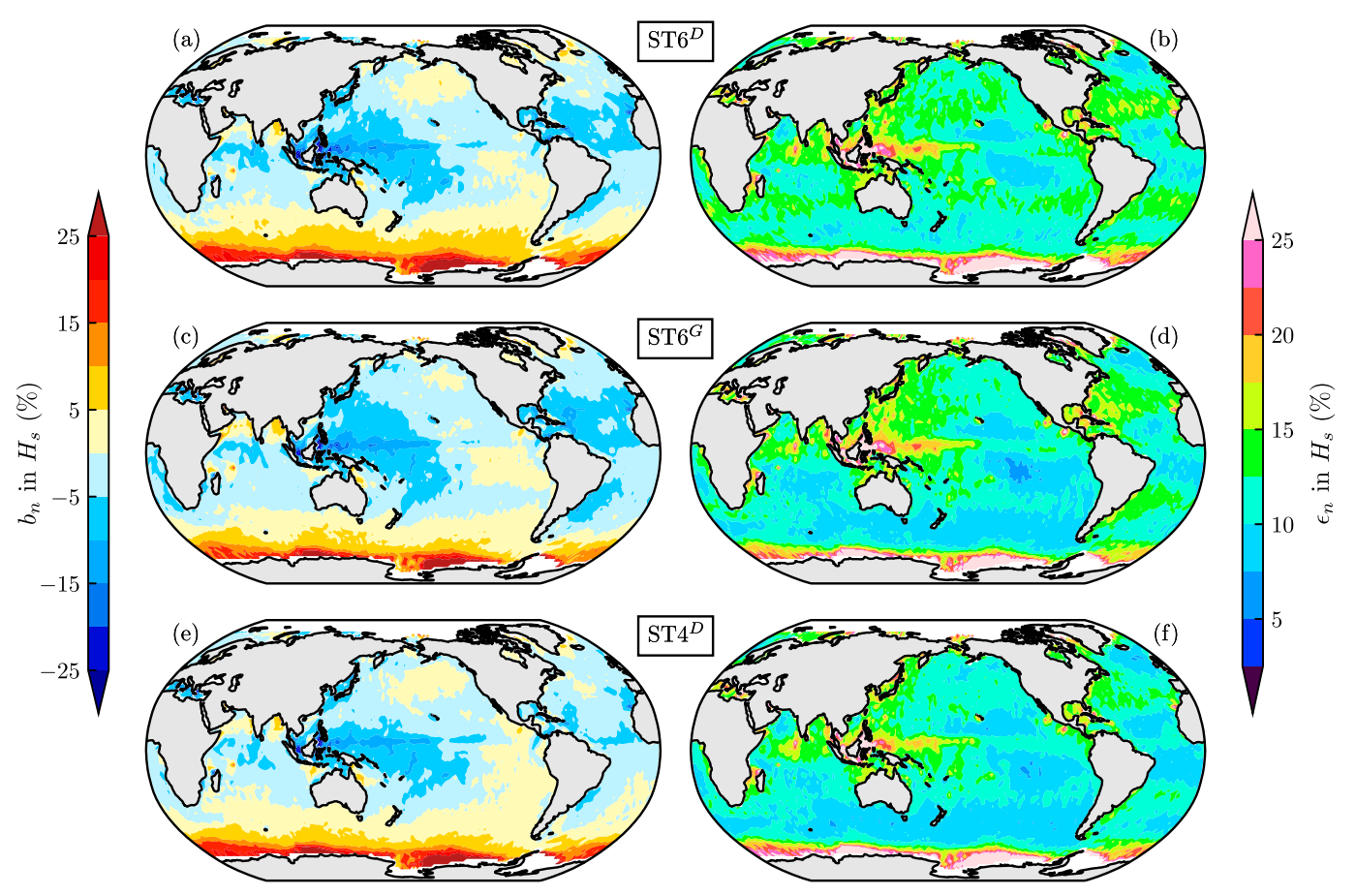

FIG. 12. Error metrics of $H_{s}$ gridded in $2^{\circ} \times 2^{\circ}$ bins for the 2011 global hindcast according to (a),(b) ST6 ${ }^{D}$, (c),(d) ST6 ${ }^{G}$, and (e),(f) ST4 ${ }^{D}$, showing (left) normalized bias $b_{n}$ and (right) normalized RMSE $\epsilon_{n}$. 


\section{b. Comparison against NDBC buoys}

The validation of model simulations against in situ buoys was also conducted using observations from a total of 21 stations (Fig. 13) maintained by the U.S. National Data Buoy Center (NDBC; http://www. ndbc.noaa.gov). Closely following SABZ16, we only selected wave buoys that provide two-dimensional wave spectra and that are located in deep water (depth $d>300 \mathrm{~m}$ ).

Figure 14 displays Taylor diagrams (Taylor 2001) summarizing the statistical comparison between wave models and buoys. Apart from wave parameters scrutinized by SABZ16 (e.g., wave height $H_{s}$, wave period $T_{0,2}$, mean square slope $\left\langle s^{2}\right\rangle$, directional spreading $\sigma_{\theta}$; see appendix A for definitions), we include another three complementary wave quantities, namely the wave period $T_{0,-1}$ (appendix $\mathrm{A}$ ), the narrowness of onedimensional spectrum $F_{n}$ [(30)], and the partial significant wave height $H_{s, i}$ (Rogers and Wang 2007; Li and Holt 2009), given by

$$
H_{s, i}=4 \sqrt{\int_{f_{l, i}}^{f_{u, i}} \int_{0}^{2 \pi} F(f, \theta) d f d \theta},
$$

where $f_{l, i}$ and $f_{h, i}$ are lower and upper integral limits. Similar to Li and Holt (2009), we divided the overlapping frequency range of buoys and models (i.e., $[0.037,0.485] \mathrm{Hz}$ ) into four bands with band boundaries locating at $f_{u, i}=0.06,0.09,0.20 \mathrm{~Hz}, i=1,2,3$. Based on a 10-day duration hindcast for Lake Michigan, Rogers and van Vledder (2013) clearly demonstrated that wave models using DIA for $S_{\mathrm{n} 1}$ tend to overestimate energy below the spectral peak and underspecify spectral narrowness and that using WRT for $S_{\mathrm{nl}}$ instead of DIA could significantly alleviate such model biases. Therefore, examinations of $H_{s, i}$ and $F_{n}$ could be beneficial to identify possible advantages of GMD over DIA. Similarly, the wave period $T_{0,-1}$ is especially relevant for the low-frequency part of the wave spectrum, and thus an evaluation of this specific period may also be informative. The Taylor diagram is not applicable to vector quantities. To give a rough idea of model skills in computing wave direction, we also plotted zonal and meridional wave heights $\left(H_{s, z}, H_{s, m}\right)=$ $H_{s}\left(\cos \theta_{w}, \sin \theta_{w}\right)$, defined in a similar fashion as zonal and meridional winds $\left(U_{10, z}, U_{10, m}\right)=U_{10}\left(\cos \theta_{u}, \sin \theta_{u}\right)$, where $\theta_{u}$ and $\theta_{w}$ are wind direction and mean wave direction (appendix A), respectively. It may be also useful to mention that the mean square slope $\left\langle s^{2}\right\rangle$ used here is actually a low-pass mean square slope as we are just looking at the waves in the buoy frequency range $(f<0.485 \mathrm{~Hz})$.

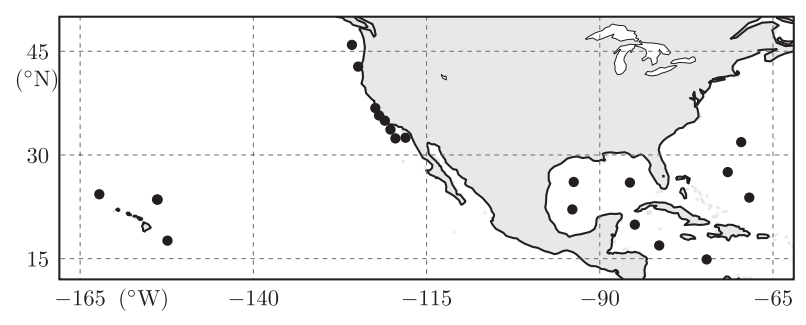

FIG. 13. A total of 21 NDBC buoys (filled circles) used in the model-buoy comparison.

Our Fig. 14 suggests that wave height $H_{s}$, wave periods $T_{0,2}$ and $T_{0,-1}$, mean square slope $\left\langle s^{2}\right\rangle$, and zonal wave height $H_{s, z}$ are generally well estimated by all three wave models, corresponding to a correlation coefficient $\rho$ greater than 0.9 , a normalized standard deviation between 0.85 and 1.1, and a normalized centered RMSE $\epsilon_{n}^{c}$ less than 0.5. Overall, the simulated $H_{s}$ is around $6 \%$ biased low at these selected buoy locations, consistent with the model-altimeter comparison shown in our Fig. 12. Wave energy at frequencies beyond $0.06 \mathrm{~Hz}$ (i.e., $H_{s, 2}, H_{s, 3}$, and $H_{s, 4}$ in Fig. 14) is simulated with a remarkably high accuracy as well. Surprisingly, model skills in estimating meridional wave height $H_{s, m}$ is appreciably inferior to those for zonal wave height $H_{s, z}$, as indicated by a lower $\rho$ and higher $\epsilon_{n}^{c}$. By contrast, error metrics for zonal and meridional winds $\left(U_{10, z}, U_{10, m}\right.$; see white and gray stars in Fig. 14a) are not that noticeably different. Interestingly, $\mathrm{ST}^{G}{ }^{G}$ indeed provides more accurate $T_{0,-1} \quad\left(\epsilon_{n}=9 \%, \rho=0.94\right)$ as compared with $\mathrm{ST}^{D}$ $\left(\epsilon_{n}=12 \%, \rho=0.92\right)$ and ST4 ${ }^{D}\left(\epsilon_{n}=11 \%, \rho=0.93\right)$.

The directional spreading $\sigma_{\theta}$ and spectral narrowness $F_{n}$ are the two most poorly simulated quantities. Both of these wave parameters show a $\epsilon_{n}^{c}$ within $[0.8,0.9]$. The $\rho$ of simulated $\sigma_{\theta}$ is about $0.7-0.75$, and the $\rho$ for $F_{n}$ is even lower $(\sim 0.5)$. The variability of $\sigma_{\theta}$ is overestimated by around $20 \%$; in contrast, models underestimate the variability of $F_{n}$ more than $35 \%$. In terms of correlation coefficient $\rho, \mathrm{ST}^{G}$ yields a slightly improved estimation of $\sigma_{\theta}\left(\rho=0.75\right.$ for ST $6^{G}$ vs $\rho=0.72,0.70$ for ST $6^{D}$ and ST $4^{D}$, respectively) and $F_{n}\left(\rho=0.55\right.$ for ST6 ${ }^{G}$ vs $\rho=0.48$ for ST6 ${ }^{D}$ and ST $\left.4^{D}\right)$. The DIA is known to overpredict directional spreading of wave spectrum (see, e.g., Fig. 9a vs Figs. 9b,c). When compared with buoy observations, model spectra however turn out to be slightly too narrow, indicated by the negative $b_{n}\left(-7 \%\right.$ and $-5 \%$ for ST $6^{D}$ and ST $\left.4^{D}\right)$. As anticipated, $\sigma_{\theta}$ from ST6 ${ }^{G}$ is more biased $(-12 \%)$. The underestimation of spreading might be attributed to inherent errors in directional distribution of different source functions and the neglect of effects of currents on waves, as already pointed out by SABZ16. 

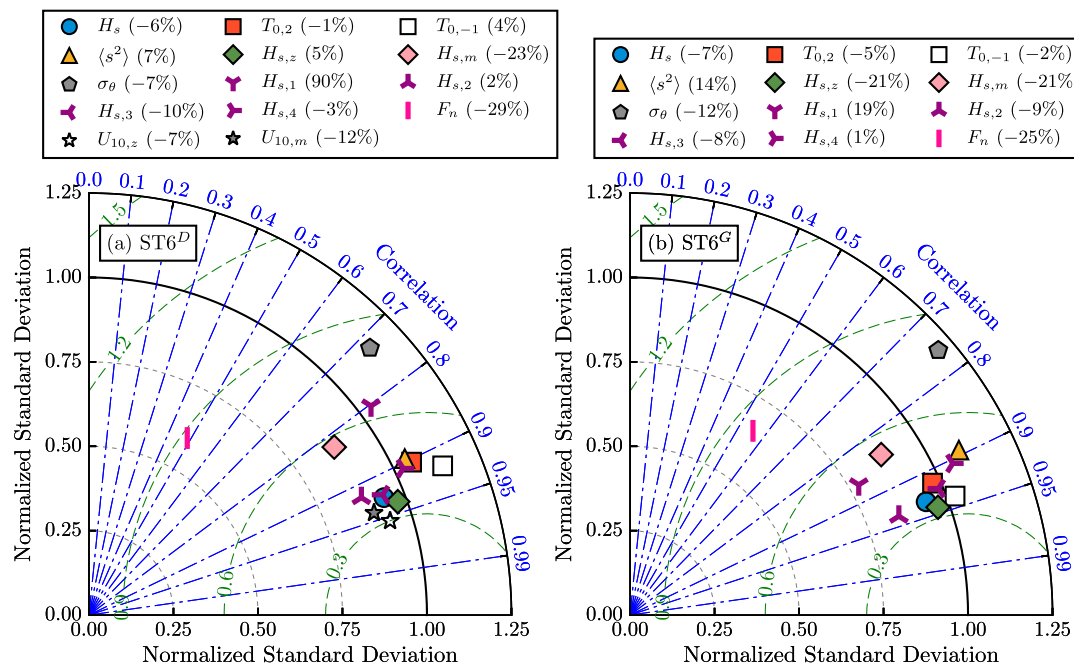
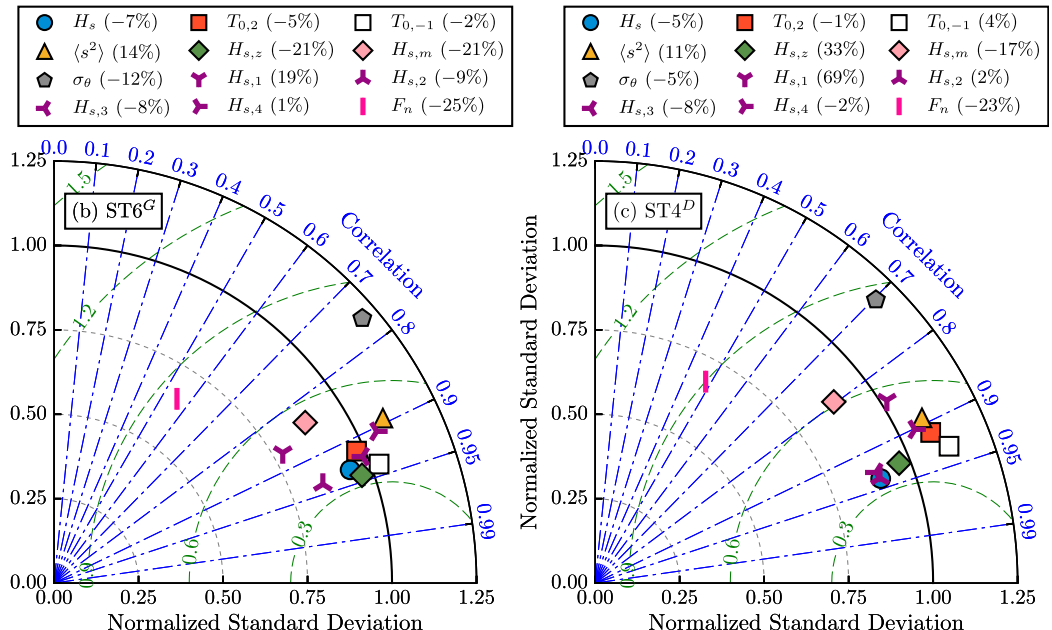

FIG. 14. Taylor diagram summarizing the statistical comparison between NDBC buoys and wave models: (a) ST6 ${ }^{D}$, (b) ST6 ${ }^{G}$, and (c) ST $4^{D}$. The wave parameters represented by different colored-markers are interpreted in figure legends. Values in parentheses identify the normalized bias $b_{n}$ of each wave parameter. The last two variables $U_{10, z}$ and $U_{10, m}$ in (a), shown as white and gray stars, illustrate comparisons of zonal and meridional winds between CFSv2 and NDBC buoys.

Numerical simulations (e.g., Fan et al. 2009a,b; Komen et al. 1994, p. 359), laboratory experiments (Rapizo et al. 2016), and field observations (Romero et al. 2017) have shown that ocean currents could broaden wave spectrum due to their refraction and scattering of wave rays. A detailed analysis of the impact of ocean currents on directionality of ocean waves will be pursued in the future. In line with Rogers and van Vledder (2013), a more accurate parameterization of $S_{\mathrm{nl}}$ is helpful for reducing the negative bias in spectral narrowness $F_{n}$ given by DIA $\left(b_{n}=-29 \%\right.$ for ST $6^{D}$ vs $b_{n}=-25 \%$ for ST6 $\left.{ }^{G}\right)$. The improvement in modeling $F_{n}$ shown here, however, is considerably less than that reported in Rogers and van Vledder (2013).

The most noticeable advantage of GMD over DIA is seen in comparisons of long-period wave energy $H_{s, 1}$ $\left(f_{u, 1}=0.06 \mathrm{~Hz}\right.$ or equivalently wave period $\left.T>16.6 \mathrm{~s}\right)$. Clearly from Fig. 14, the accuracy of modeled $H_{s, 1}$ is not as high as that for the three other counterparts $\left(H_{s, 2}\right.$, $H_{s, 3}$, and $H_{s, 4}$ ). ST6 ${ }^{D}$ (Fig. 14a) exhibits a normalized bias $b_{n}$ of $90 \%$ for $H_{s, 1}$, indicating a serious overestimation of energy at frequencies below $0.06 \mathrm{~Hz}$. ST $4^{D}$ (Fig. 14c) provides a somewhat improved performance in specifying $H_{s, 1}\left(b_{n}=69 \%\right)$. This large positive bias is significantly reduced in the $\mathrm{ST}^{G}{ }^{G}$ simulation, as corroborated by a much lower $b_{n}(19 \%)$. The variability of $H_{s, 1}$ is underestimated by ST6 ${ }^{G}$ by around $20 \%$.

To highlight improvements in simulating high-frequency energy brought about by the recalibrated $\mathrm{ST}^{D}{ }^{D}$ over its predecessor (ZBRY15), and improvements in modeling low-frequency energy brought about by use of the GMD over the DIA, we replotted the mean square slope $\left\langle s^{2}\right\rangle$ and partial wave height $H_{s, i}$ in Figs. 15 and 16, respectively. Similar to Ardhuin et al. (2010) and SABZ16, Fig. 15 illustrates the averaged mean square slope $\left\langle s^{2}\right\rangle$ over each $1 \mathrm{~m} \mathrm{~s}^{-1}$ bin of $U_{10}$ and each $0.5 \mathrm{~m}$ bin of $H_{s}$. SABZ16 (their Fig. 7) reported that the predecessor of $\mathrm{ST}^{D}$ (ZBRY15) was inclined to overestimate $\left\langle s^{2}\right\rangle$, particularly under high winds. For instance, for $U_{10}=$ $15 \mathrm{~m} \mathrm{~s}^{-1}$ and $H_{s} \sim 4.5 \mathrm{~m}$, the old version of ST6 ${ }^{D}$ overestimated $\left\langle s^{2}\right\rangle$ by more than $30 \%$. Similar to ST4 ${ }^{D}$ (Fig. 15d), the recalibrated ST6 ${ }^{D}$ and ST6 ${ }^{G}$ (Figs. 15b,c) reproduce the variability of $\left\langle s^{2}\right\rangle$ with $U_{10}$ and $H_{s}$ as measured by buoys (Fig. 15a) remarkably well. The scatter density plots of $H_{s, i}$ shown in Fig. 16 provide a more intuitive visualization of the decrease of model errors in calculating $H_{s, 1}$ by ST6 ${ }^{G}$ relative to that for the DIA-based simulations (Figs. 16a-c). All the four error metrics (i.e., $b, \epsilon, \rho$, and SI) for $H_{s, 1}$ provided by $\mathrm{ST}^{G}$ are slightly better than those from $\mathrm{ST}^{D}$ and ST4 ${ }^{D}$. As already mentioned, this improvement is consistent with what we expect from the findings of Rogers and van Vledder (2013). It is worth noting that the remaining marginally positive bias $b_{n}=0.03 \mathrm{~m}$ of $H_{s, 1}$ in the ST6 ${ }^{G}$ simulation can be further reduced when coastlines and small islands are resolved better, as demonstrated in Li (2012, his Fig. 5).

One detail that needs to be further clarified is that the swell decay coefficient $B_{1}$ in (12) for ST6 ${ }^{G}$ is slightly higher than that used for $\operatorname{ST}^{D}\left(6.0 \times 10^{-3}\right.$ vs $4.1 \times 10^{-3}$; Table 1). One may argue that the reduction of positive bias in $H_{s, 1}$ by $\mathrm{ST}^{G}$ might be attributed to the higher $B_{1}$. Another global hindcast of 2011 by ST6 $6^{D}$ but using $a_{0}$ and $B_{1}$ from ST6 $^{G}$ (i.e., $a_{1}=0.05$ and $B_{1}=6.0 \times 10^{-3}$ ) 

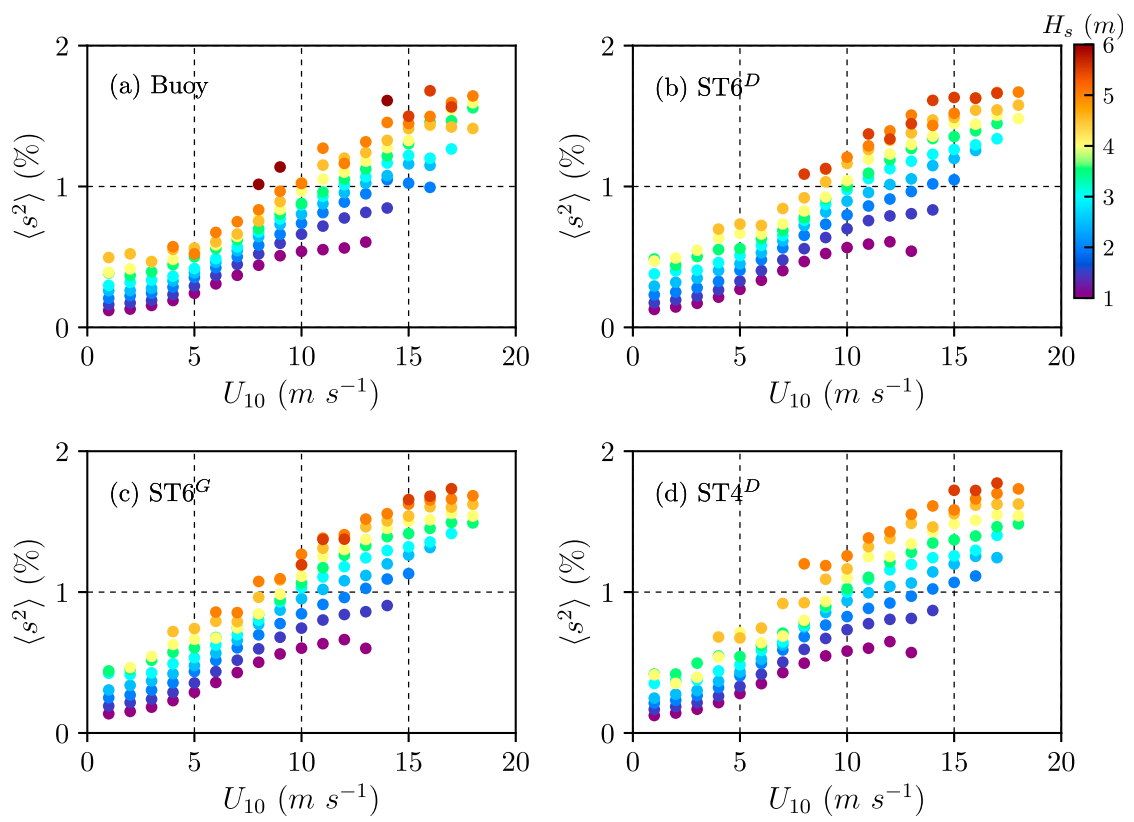

FIG. 15. Averaged mean square slope $\left\langle s^{2}\right\rangle$ in each $1 \mathrm{~m} \mathrm{~s}^{-1}$ bin of $U_{10}$ and each $0.5 \mathrm{~m}$ bin of $H_{s}$ according to (a) NDBC buoys, (b) ST6 ${ }^{D}$, (c) ST6 ${ }^{G}$, and (d) ST4 $4^{D}$.

corresponds to a $b_{n}$ in $H_{s, 1}$ of $78 \%$ (section 6 of the SOM), which is still considerably higher than that for $\mathrm{ST}^{G}(19 \%)$. Therefore, the advantage of ST6 ${ }^{G}$ over $\mathrm{ST}^{D}$ in estimating low-frequency wave energy is a robust feature.

Having seen the marked advantage of GMD over DIA in estimating low-frequency wave energy, we expect that GMD might also be advantageous in characterizing different wave systems, at least for low-frequency swells. This is checked in Fig. 17, where Taylor diagrams for partitioned wave parameters are displayed. The watershed algorithm (Hanson and Phillips 2001) combined with a digital filter proposed by Portilla et al. (2009) was utilized to identify distinct partitions from two-dimensional wave spectrum $F(f, \theta)$. Following Hanson and Phillips (2001), partitions with $c_{p} / U_{10} \cos \theta_{u p} \leq 1.5$ were considered as wind seas and swells otherwise, where $\theta_{u p}$ is the angle between wind $\theta_{u}$ and partition peaks $\theta_{p}$. Following Delpey et al. (2010) and SABZ16, another criterion

$$
\begin{aligned}
d_{n}= & \| f_{p}^{o} \cos \theta_{p}^{o}-f_{p}^{m} \cos \theta_{p}^{m}, f_{p}^{o} \sin \theta_{p}^{o} \\
& -f_{p}^{m} \sin \theta_{p}^{m} \| / f_{p}^{o} \leq 0.3
\end{aligned}
$$

was successively applied to match partitions from buoys and models, where $d_{n}$ is the normalized Euclidean distance between the partition peak of the buoys $\left(f_{p}^{o}, \theta_{p}^{o}\right)$ and that of the models $\left(f_{p}^{m}, \theta_{p}^{m}\right)$. More than 130000 matchups are located between buoys and each of three wave models, among which the proportion of wind seas is strikingly low $(\sim 18 \%)$. This is, however, comparable to the frequency of occurrence of pure wind sea at buoy locations as presented in Hanley et al. (2010, their Fig. 9). Inspection of Fig. 17 suggests that model skills in estimating wind-sea parameters (markers with outlines) are higher than those for swell, with the only exception being the peak wave period $T_{p}$. As for Fig. 14, the directional spreading $\sigma_{\theta}$ and spectral narrowness $F_{n}$ are the most poorly resolved quantities. The wave models have no skills for $\sigma_{\theta}$ of swells. Contrary to our expectation, ST6 ${ }^{G}$ (Fig. 17b) only shows marginally improved performance in representing different wave systems as compared against ST $6^{D}$ and ST4 ${ }^{D}$ (Figs. 17a,c; Table 3).

The last problem to be addressed here is the computational efficiency of different wave models. Table 4 shows the normalized model run times obtained with wave models using different nonlinear solvers and different prognostic frequency ranges. The high-frequency limit $f_{h, f}$ of the prognostic region of the spectrum is defined by $f_{h, f}=N_{h, f} / T_{0,-1}$ (appendix A), where $N_{h, f}$ is a real number and $N_{h, f} \geq 2.5$ is generally used in the literature (e.g., Hasselmann et al. 1988). Consistent with ZBRY $15, \mathrm{ST}^{D}{ }^{D}$ is about $40 \%$ more expensive than $\mathrm{ST}^{D}$. ST6 ${ }^{G}$ is around 5.6 times as expensive as $\mathrm{ST}^{D}$, indicating that the GMD configuration with five quadruplets and a three-parameter quadruplet definition might not be 

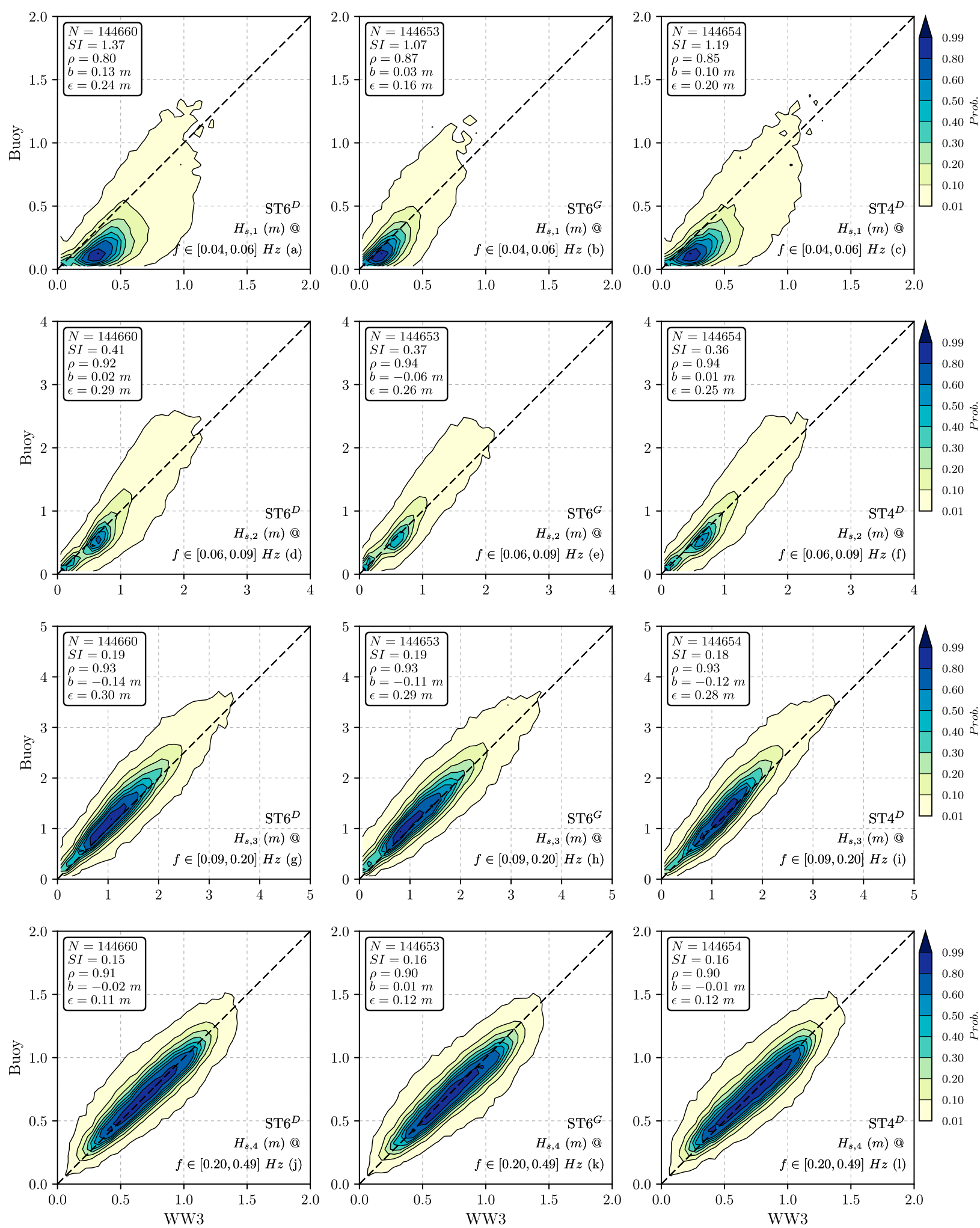

FIG. 16. Comparison of partial wave heights for (top) $H_{s, 1}$, (top middle) $H_{s, 2}$, (bottom middle) $H_{s, 3}$, and (bottom) $H_{s, 4}$ between NDBC buoys and wave models: (left) ST6 ${ }^{D}$, (center) ST6 ${ }^{G}$, and (right) ST4 ${ }^{D}$. The lower and upper limits $f_{l, i}$ and $f_{h, i}[(36)]$ are printed in the lowerright corner of each panel. 

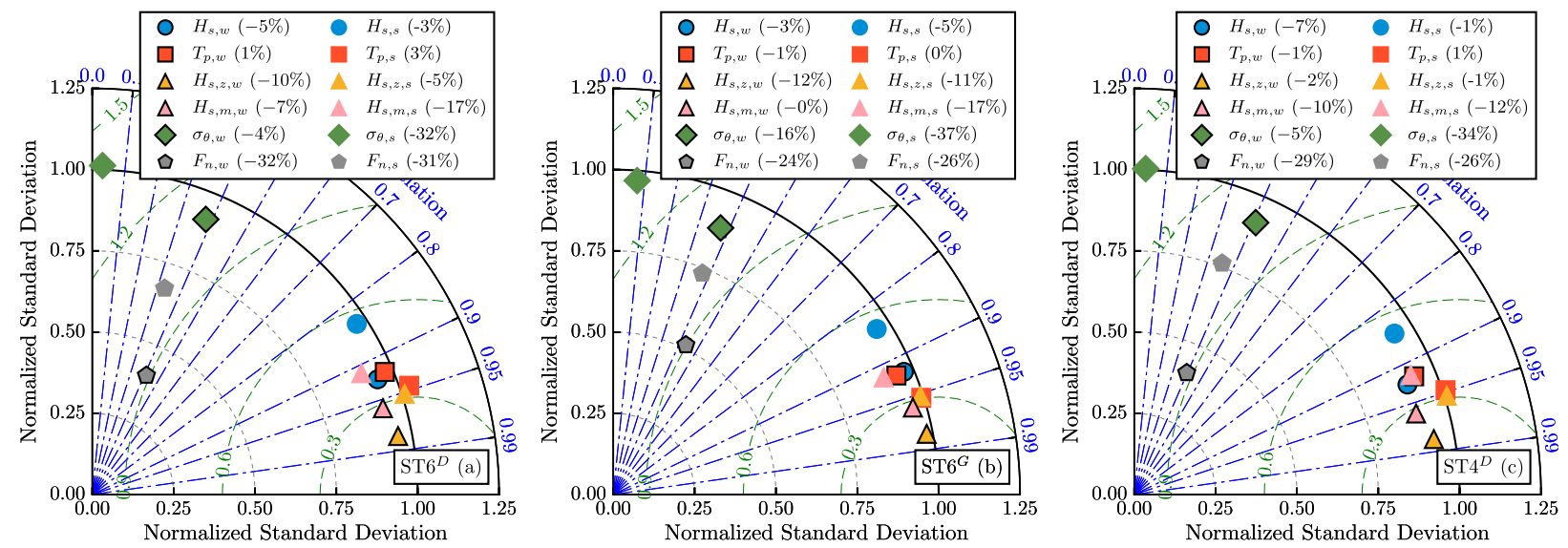

FIG. 17. As in Fig. 14, but for partitioned wave parameters from (a) $\mathrm{ST}^{D}$, (b) $\mathrm{ST}_{6}^{G}$, and (c) $\mathrm{ST} 4^{D}$. The last subscripts $w$ and $s$ of variables printed in figure legends denote parameters for wind sea (markers with black outlines) and swell (markers without black outlines), respectively.

economically feasible for operational purpose (see also Tolman 2013). The GMD approach with less complex configurations, as described in Tolman (2013) and Tolman and Grumbine (2013), should be pursued for operational research.

\section{Concluding remarks}

In this paper, the observation-based source term package in WW3, that is, the ST6 for $S_{\text {in }}+S_{\mathrm{ds}}+S_{\mathrm{wl}}$ (RBW12; ZBRY15), is recalibrated and verified through a series of academic and realistic simulations, including the fetch/duration-limited test, a Lake Michigan (no swell) hindcast, and a 1-yr global hindcast. In addition to the traditional bulk (integral) criteria, we introduced spectral metrics for model validations. We also specifically optimized the GMD nonlinear solver for ST6 (Fig. 1) in order to investigate the DIA-induced uncertainties in spectral wave modeling. Key findings are summarized below:

1) The updated $\mathrm{ST}^{D}$ (i.e., ST6 + DIA) and $\mathrm{ST}^{G}$ (i.e., ST6 + GMD) not only are skillful in simulating commonly used bulk wave parameters (e.g., $H_{s}$ and wave periods) but also accurately represent the high-frequency wave spectrum [in terms of the saturation spectrum $B(f)$ and mean square slope $\left.\left\langle s^{2}\right\rangle\right]$. The overestimation of high-frequency wave energy by ST6's predecessor (e.g., ZBRY15) is resolved by the recalibration described here (e.g., Fig. 15).

2) In the duration-limited test, $E(f)$ simulated by ST6 models $\left[\mathrm{ST}^{D}{ }^{D}, \mathrm{ST}^{G}{ }^{G}\right.$, and ST6 ${ }^{W}$ (i.e., ST6 + WRT); Figs. $4 \mathrm{a}, \mathrm{c}, \mathrm{e}]$ shows a clear transition behavior from the power law of approximately $f^{-4}$ to the power law of approximately $f^{-5}$. The modeled energy level of the equilibrium range $\left[E(f) \cong f^{-4}\right]$, as represented by the wave age-dependent Toba's parameter $\alpha_{T}$, is in good agreement with field measurements from Hwang et al. (2000a) and Resio et al. (2004) (Fig. 5). The saturation level $B(f)$ yielded by these three ST6 models is also consistent with observations from Babanin and Soloviev (1998a), RM10, and Lenain and Melville (2017) (Figs. 4b,d,f). In addition, the ST6-predicted transition frequency $f_{t}$ from an $E(f) \propto f^{-4}$ to $E(f) \stackrel{\propto}{\sim} f^{-5}$ is comparable to buoy data from Forristall (1981).

3) The wave spectra from $\mathrm{ST}^{G}$ are in excellent agreement with those from ST6 ${ }^{W}$, particularly in the frequency space (Figs. 4-9), illustrating the high accuracy of the GMD approach in reproducing exact solutions of $S_{\mathrm{nl}}$ from WRT. In the global hindcast, ST $6^{G}$ exhibits a much better performance in predicting low-frequency wave energy. The normalized biases of $H_{s, 1}$ (wave period $T>16.6 \mathrm{~s}$ ) given by $\mathrm{ST}^{D}$ and $\mathrm{ST} 4^{D}$ are $90 \%$ and $69 \%$, respectively, whereas such model errors are significantly reduced by $\mathrm{ST}^{G}\left(b_{n}=19 \%\right)$ (Figs. 14 and 16), which is analogous to the findings from Rogers and van Vledder (2013). Nonetheless, contrary to our expectation, $\mathrm{ST} 6^{G}$ only provides marginal improvement in characterizing different wave systems (i.e., wind sea and swell; Fig. 17). The GMD configuration used here is $\sim 5$ times more expensive than the DIA (Table 4) and therefore might not be economically feasible for operational forecasting.

4) When we fit the generalized JONSWAP spectrum (28) to the modeled $E(f)$ from the duration-limited 
TABLE 3. Statistical comparison (as represented by the normalized RMSE $\epsilon_{n}$ and correlation coefficient $\rho$ ) of partitioned wave parameters between NDBC buoys and wave models. The normalized bias $b_{n}$ is presented in Fig. 17 and thus is not reproduced here.

\begin{tabular}{|c|c|c|c|c|c|c|c|c|c|c|c|c|}
\hline \multirow[b]{2}{*}{ Wind sea } & \multicolumn{2}{|r|}{$H_{s, w}$} & \multicolumn{2}{|c|}{$T_{p, w}$} & \multicolumn{2}{|c|}{$H_{s, z, w}$} & \multicolumn{2}{|c|}{$H_{s, m, w}$} & \multicolumn{2}{|c|}{$\sigma_{\theta, w}$} & \multicolumn{2}{|c|}{$F_{n, w}$} \\
\hline & & $\rho$ & $\epsilon_{n}$ & $\rho$ & $\epsilon_{n}$ & $\rho$ & $\epsilon_{n}$ & $\rho$ & $\epsilon_{n}$ & $\rho$ & $\epsilon_{n}$ & $\rho$ \\
\hline $\mathrm{ST} 6^{D}$ & & 0.93 & 0.09 & 0.92 & 0.18 & 0.98 & 0.26 & 0.96 & 0.17 & 0.38 & 0.40 & 0.41 \\
\hline $\mathrm{ST} 6^{G}$ & & 0.92 & 0.10 & 0.92 & 0.19 & 0.98 & 0.25 & 0.96 & 0.23 & 0.37 & 0.35 & 0.44 \\
\hline \multirow[t]{2}{*}{$\mathrm{ST} 4^{D}$} & & 0.93 & 0.09 & 0.92 & 0.18 & 0.98 & 0.26 & 0.96 & 0.18 & 0.41 & 0.38 & 0.40 \\
\hline & \multicolumn{2}{|c|}{$H_{s, s}$} & \multicolumn{2}{|c|}{$T_{p, s}$} & \multicolumn{2}{|c|}{$H_{s, z, s}$} & \multicolumn{2}{|c|}{$H_{s, m, s}$} & \multicolumn{2}{|c|}{$\sigma_{\theta, s}$} & \multicolumn{2}{|c|}{$F_{n, s}$} \\
\hline Swell & $\epsilon_{n}$ & $\rho$ & $\epsilon_{n}$ & $\rho$ & $\epsilon_{n}$ & $\rho$ & $\epsilon_{n}$ & $\rho$ & $\epsilon_{n}$ & $\rho$ & $\epsilon_{n}$ & $\rho$ \\
\hline $\mathrm{ST} 6^{D}$ & 0.28 & 0.84 & 0.10 & 0.95 & 0.30 & 0.95 & 0.38 & 0.91 & 0.48 & 0.03 & 0.43 & 0.33 \\
\hline $\mathrm{ST} 6^{G}$ & 0.27 & 0.85 & 0.09 & 0.95 & 0.30 & 0.95 & 0.37 & 0.92 & 0.50 & 0.07 & 0.40 & 0.37 \\
\hline $\mathrm{ST} 4^{D}$ & 0.27 & 0.85 & 0.10 & 0.95 & 0.30 & 0.95 & 0.37 & 0.92 & 0.49 & 0.03 & 0.40 & 0.35 \\
\hline
\end{tabular}

case, the simulated high-frequency $\alpha$ is generally consistent with previous field studies (e.g., Hasselmann et al. 1976; Donelan et al. 1985). The simulated spectral peakedness, in terms of $\gamma[(28)]$ or width $F_{w}$ [(29)], however, generally deviates from field observations (e.g., Donelan et al. 1985; Krivinskii 1991; Babanin and Soloviev 1998a) (Figs. 7 and 8). In particular, the peak of $E(f)$ from $\mathrm{ST}^{G}$ and $\mathrm{ST}^{w}$ appears too narrow, consistent with the finding of the recent numerical study by Annenkov and Shrira (2018).

5) A few problems presented here still remain unsolved, including that 1) the spectral narrowness $F_{n}$ and directional spreading $\sigma_{\theta}$ are quite poorly resolved in the global simulations, as shown in Figs. 14 and 17;2) wave models are able, to some degree, to present bimodal structure of short waves (Fig. 10); the lobe ratio $r_{\text {lobe }}$, however, is considerably underestimated (SOM); and 3) the model bias in the Southern Ocean is still relatively high $\left(b_{n} \sim 10 \%\right)$. All these issues are left for future research.

Finally, we conclude this study with the following recommendations:

1) $\mathrm{ST}_{6}{ }^{D}$ and $\mathrm{ST} 4^{D}$ provide good, and very close, performance in estimating the commonly used integral wave parameters (significant wave height $H_{s}$, wave periods, mean square slope $\left\langle s^{2}\right\rangle$ etc.), and therefore either of the two is applicable to the operational wave forecasting and hindcasting. Perrie et al. (2018) demonstrated that in their highresolution wave forecast model systems, ST $4^{D}$ outperformed the physics package originally designed for the WAM model (Hasselmann et al. 1988) but at the expense of at least $50 \%$ more CPU time. Considering the slightly higher computational efficiency of $\mathrm{ST}^{D}$ in the $1-\mathrm{yr}$ global hindcast (Table 4), we expect that $\mathrm{ST}^{D}$ may save noticeable computational costs in such refined, highresolution applications.

2) The wave spectrum of short gravity waves is crucial to estimate the wave-induced momentum flux from wind (e.g., Chalikov and Rainchik 2011). Since ST6 yields an improved high-frequency tail, it is recommended to further test/verify this package in the fully coupled atmosphere-wave-ocean models (e.g., Fan et al. 2009a; Warner et al. 2010; Chen et al. 2013).

3) The $\mathrm{ST}^{G}{ }^{G}$ model configuration (five quadruplets) increases computational costs by a factor of about 6 , restricting its applicability to research purposes only, at least at this stage. For academic studies particularly concerned with low-frequency wave energy, $\mathrm{ST} 6^{G}$ is preferred over $\mathrm{ST}^{D}$.

4) Only deep-water waves are considered in our present study. A thorough validation of the updated ST6 configurations in the finite-deep and shallow waters, similar to the work conducted by Aijaz et al. (2016) and van Vledder et al. (2016), is recommended for further analyses.

TABLE 4. The normalized model run time for 1-month global hindcast using the model setup summarized in section 5 . The highfrequency limit $f_{h, f}$ of the prognostic region of the spectrum is defined by $f_{h, f}=N_{h, f} / T_{0,-1}$ (appendix A), where $N_{h, f}=-$ means the high-frequency spectral tail evolves freely without any prescribed slope. All the run times are normalized with the run time obtained with ST6 ${ }^{D}$ and $N_{h, f}=6$.

\begin{tabular}{ccc}
\hline \hline Model & $N_{h, f}$ & Time \\
\hline $\mathrm{ST}^{D}$ & 6 & 1.00 \\
$\mathrm{ST}^{D}$ & - & 1.16 \\
$\mathrm{ST}^{G}$ & 6 & 5.68 \\
$\mathrm{ST}^{G}$ & - & 6.55 \\
$\mathrm{ST}^{D}$ & 6 & 1.39 \\
\hline
\end{tabular}


Acknowledgments. The authors are grateful to Dr. Kevin Ewans from MetOcean Research Ltd, New Zealand, for discussion about various aspects of wave spectra. The distribution and maintenance of WW3 and GMD codes by NOAA/NCEP is gratefully acknowledged. We appreciate Dr. David W. Wang from the U.S. Naval Research Laboratory for providing his code of the Maximum Entropy Method (MEM). QL, AVB, and IRY acknowledge the support from the DISI AustraliaChina Centre through Grant ACSRF48199. AVB also appreciates the financial support by ARC Discovery DP170101328. FQ is supported by the international cooperation project on the China-Australia Research Centre for Maritime Engineering of Ministry of Science and Technology, China under Grant 2016YFE0101400. We thank Dr. G. P. van Vledder and another reviewer for their detailed comments and suggestions that have improved our manuscript a lot.

\section{APPENDIX A}

\section{Integral Parameters from the Wave Spectrum}

The bulk parameters selected to evaluate wave model skills, including significant wave height $H_{s}$, mean wave period $T_{0,2}$, mean square slope $\left\langle s^{2}\right\rangle$, mean wave direction $\theta_{w}$, and directional spreading $\sigma_{\theta}$, are calculated from the two-dimensional wave spectrum $F(f, \theta)$ as follows (see, e.g., T16 and Kuik et al. 1988):

$$
\begin{aligned}
m_{n} & =\int_{f_{l}}^{f_{u}} \int_{0}^{2 \pi} f^{n} F(f, \theta) d f d \theta, \\
H_{s} & =4 \sqrt{m_{0}}, \\
T_{0,2} & =\sqrt{m_{0} / m_{2}}, \\
T_{0,-1} & =m_{-1} / m_{0}, \\
\left\langle s^{2}\right\rangle & =\int_{f_{l}}^{f_{u}} \int_{0}^{2 \pi} k^{2} F(f, \theta) d f d \theta, \\
a & =\int_{f_{l}}^{f_{u}} \int_{0}^{2 \pi} \cos \theta F(f, \theta) d f d \theta, \\
b & =\int_{f_{l}}^{f_{u}} \int_{0}^{2 \pi} \sin \theta F(f, \theta) d f d \theta, \\
\theta_{w} & =\arctan (b / a), \\
\sigma_{\theta} & =\sqrt{2\left[1-\sqrt{\left(a^{2}+b^{2}\right) / m_{0}^{2}}\right],}
\end{aligned}
$$

where $f_{l}$ and $f_{u}$ are the lower and upper limits of integration. When wave models are verified against buoy observations, $f_{l}=0.037 \mathrm{~Hz}$ and $f_{u}=0.485 \mathrm{~Hz}$ are adopted. The peak period $T_{p}=1 / f_{p}$ is calculated from the omnidirectional frequency spectrum $E(f)$ using a

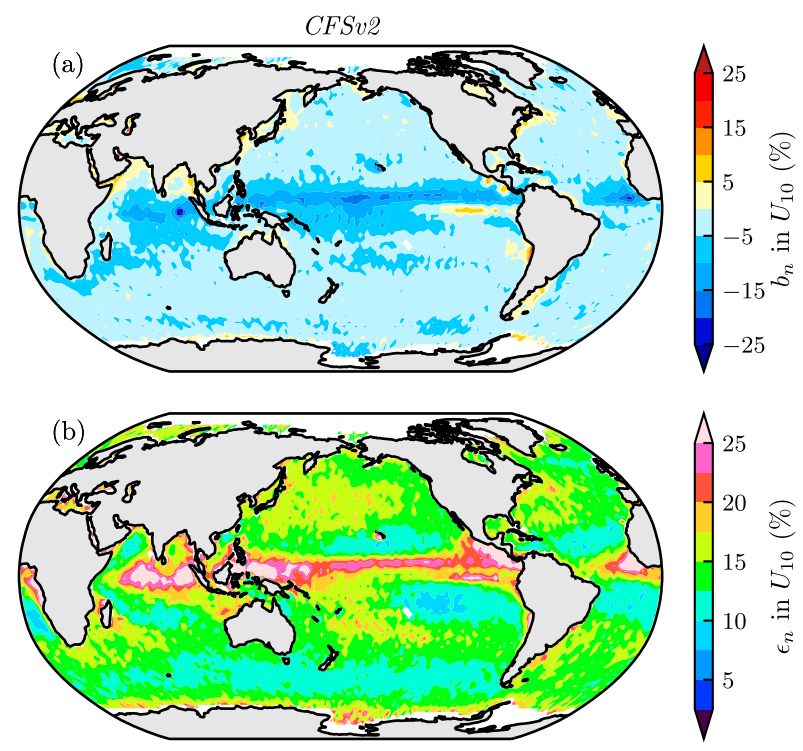

FIG. B1. As in Fig. 12, but for (a) the normalized bias $b_{n}$ and (b) normalized RMSE $\epsilon_{n}$ of CFSv2 $U_{10}$, showing 2011 relative to altimeter measurements.

parabolic fit in the neighborhood of the spectral peak. Following Ardhuin et al. (2010), the normalized bias $b_{n}$ and $\epsilon_{n}$ are

$$
\begin{aligned}
b_{n} & =\sum_{i=1}^{N}\left(x_{i}-y_{i}\right) \mid \sum_{i=1}^{N} y_{i}, \\
\epsilon_{n} & =\sqrt{\sum_{i=1}^{N}\left(x_{i}-y_{i}\right)^{2} / \sum_{i=1}^{N} y_{i}^{2},}
\end{aligned}
$$

where $x_{i}$ and $y_{i}$ are simulated and measured wave quantities, respectively. The other metrics, including bias $b$, RMSE $\epsilon$, correlation coefficient $\rho$, scatter index SI, and normalized centered RMSE $\epsilon_{n}^{c}$ are calculated by their standard definitions [see, e.g., the appendix of Liu et al. (2017)] and thus are not described here.

\section{APPENDIX B}

\section{The Performance of the CFSv2 Winds}

The performance of the CFSv2 winds of 2011 was carefully checked by SABZ16 (their section 2.3), using $U_{10}$ measurements from the Envisat altimeter as the reference. Demonstrating the remarkably high accuracy of the CFSv2 wind forcing, SABZ16 showed the spatial distribution of the normalized bias $b_{n}$ only in their Fig. 2 . Motivated by our reviewers, here we provided the error maps of both $b_{n}$ and $\epsilon_{n}$ in Fig. B1, which are helpful for explaining the wave model errors shown in Fig. 12. Altimeter $U_{10}$ observations were sourced from the 
multiple altimeter dataset (Young et al. 2017), and four altimeters (Jason-1/2, Envisat, and CryoSat-2) were selected.

\section{REFERENCES}

Aijaz, S., W. E. Rogers, and A. V. Babanin, 2016: Wave spectral response to sudden changes in wind direction in finite-depth waters. Ocean Modell., 103, 98-117, https://doi.org/10.1016/ j.ocemod.2015.11.006.

Alves, J. H. G. M., and M. L. Banner, 2003: Performance of a saturation-based dissipation-rate source term in modeling the fetch-limited evolution of wind waves. J. Phys. Oceanogr., 33, 1274-1298, https://doi.org/10.1175/1520-0485(2003)033<1274: POASDS $>2.0 . \mathrm{CO} ; 2$.

, - , and I. R. Young, 2003: Revisiting the PiersonMoskowitz asymptotic limits for fully developed wind waves. J. Phys. Oceanogr., 33, 1301-1323, https://doi.org/10.1175/ 1520-0485(2003)033<1301:RTPALF $>2.0 . C O ; 2$.

- S. Stripling, A. Chawla, H. L. Tolman, and A. J. van der Westhuysen, 2015: Operational wave guidance at the U.S. National Weather Service during Tropical/post-Tropical Storm Sandy, October 2012. Mon. Wea. Rev., 143, 1687-1702, https://doi.org/10.1175/MWR-D-14-00143.1.

Annenkov, S. Y., and V. I. Shrira, 2018: Spectral evolution of weakly nonlinear random waves: Kinetic description versus direct numerical simulations. J. Fluid Mech., 844, 766-795, https://doi.org/10.1017/jfm.2018.185.

Ardhuin, F., and A. D. Jenkins, 2006: On the interaction of surface waves and upper ocean turbulence. J. Phys. Oceanogr., 36, 551-557, https://doi.org/10.1175/JPO2862.1.

- B. Chapron, and F. Collard, 2009: Observation of swell dissipation across oceans. Geophys. Res. Lett., 36, L06607, https:// doi.org/10.1029/2008GL037030.

— functions for ocean waves. Part I: Definition, calibration, and validation. J. Phys. Oceanogr., 40, 1917-1941, https://doi.org/ 10.1175/2010JPO4324.1.

—, J. Tournadre, P. Queffeulou, F. Girard-Ardhuin, and F. Collard, 2011: Observation and parameterization of small icebergs: Drifting breakwaters in the Southern Ocean. Ocean Modell., 39, 405-410, https://doi.org/10.1016/ j.ocemod.2011.03.004.

Ataktürk, S. S., and K. B. Katsaros, 1999: Wind stress and surface waves observed on Lake Washington. J. Phys. Oceanogr., 29, 633-650, https://doi.org/10.1175/1520-0485(1999)029<0633: WSASWO $>2.0 . \mathrm{CO} ; 2$.

Babanin, A. V., 2006: On a wave-induced turbulence and a wavemixed upper ocean layer. Geophys. Res. Lett., 33, L20605, https://doi.org/10.1029/2006GL027308.

—_, 2010: Wind input, nonlinear interactions and wave breaking at the spectrum tail of wind-generated waves; transition from $f^{-4}$ to $f^{-5}$ behaviour. Ecological Safety of Coastal and Shelf Zones and Comprehensive Use of Shelf Resources, Vol. 21, Marine Hydrophysical Institute, 173-187.

- 2011: Breaking and Dissipation of Ocean Surface Waves. Cambridge University Press, 480 pp., https://doi.org/10.1017/ CBO9780511736162.

, and Y. P. Soloviev, 1998a: Field investigation of transformation of the wind wave frequency spectrum with fetch and the stage of development. J. Phys. Oceanogr., 28, 563-576,
https://doi.org/10.1175/1520-0485(1998)028<0563:FIOTOT $>$ 2.0.CO;2.

_- and — - 1998b: Variability of directional spectra of wind-generated waves, studied by means of wave staff arrays. Mar. Freshwater Res., 49, 89-101, https://doi.org/10.1071/ MF96126.

— and I. R. Young, 2005: Two-phase behaviour of the spectral dissipation of wind waves. Fifth Int. Symp. on Ocean Wave Measurements and Analysis (WAVES2005), Madrid, Spain, CEDEX, 51.

— , and B. K. Haus, 2009: On the existence of water turbulence induced by nonbreaking surface waves. J. Phys. Oceanogr., 39, 2675-2679, https://doi.org/10.1175/2009JPO4202.1.

_ I. R. Young, and M. L. Banner, 2001: Breaking probabilities for dominant surface waves on water of finite constant depth. J. Geophys. Res., 106, 11 659-11 676, https://doi.org/10.1029/ 2000JC000215.

_ M. M. Banner, I. R. Young, and M. A. Donelan, 2007: Wavefollower field measurements of the wind-input spectral function. Part III: Parameterization of the wind-input enhancement due to wave breaking. J. Phys. Oceanogr., 37, 2764-2775, https://doi.org/10.1175/2007JPO3757.1.

— K. N. Tsagareli, I. R. Young, and D. J. Walker, 2010: Numerical investigation of spectral evolution of wind waves. Part II: Dissipation term and evolution tests. J. Phys. Oceanogr., 40, 667-683, https://doi.org/10.1175/2009JPO4370.1.

Banner, M. L., 1990: Equilibrium spectra of wind waves. J. Phys. Oceanogr., 20, 966-984, https://doi.org/10.1175/1520-0485(1990) 020<0966:ESOWW>2.0.CO;2.

— , and I. R. Young, 1994: Modeling spectral dissipation in the evolution of wind waves. Part I: Assessment of existing model performance. J. Phys. Oceanogr., 24, 1550-1571, https:// doi.org/10.1175/1520-0485(1994)024<1550:MSDITE>2.0.CO;2.

- A. V. Babanin, and I. R. Young, 2000: Breaking probability for dominant waves on the sea surface. J. Phys. Oceanogr., 30, 3145-3160, https://doi.org/10.1175/1520-0485(2000)030<3145: BPFDWO $>2.0 . \mathrm{CO} ; 2$.

Battjes, J. A., T. J. Zitman, and L. H. Holthuusen, 1987: A reanalysis of the spectra observed in JONSWAP. J. Phys. Oceanogr., 17, 1288-1295, https://doi.org/10.1175/1520-0485(1987) $017<1288$ :AROTSO $>2.0$.CO;2.

Booij, N., R. C. Ris, and L. H. Holthuijsen, 1999: A thirdgeneration wave model for coastal regions: 1 . Model description and validation. J. Geophys. Res., 104, 7649-7666, https:// doi.org/10.1029/98JC02622.

Cavaleri, L., and Coauthors, 2007: Wave modelling-The state of the art. Prog. Oceanogr., 75, 603-674, https://doi.org/10.1016/ j.pocean.2007.05.005.

_ , and Coauthors, 2018: Wave modelling in coastal and inner seas. Prog. Oceanogr., 167, 164-233, https://doi.org/10.1016/ j.pocean.2018.03.010.

Chalikov, D., and S. Rainchik, 2011: Coupled numerical modelling of wind and waves and the theory of the wave boundary layer. Bound.-Layer Meteor., 138,1-41, https://doi.org/10.1007/ s10546-010-9543-7.

Chen, S. S., W. Zhao, M. A. Donelan, and H. L. Tolman, 2013: Directional wind-wave coupling in fully coupled atmosphere-wave-ocean models: Results from CBLASTHurricane. J. Atmos. Sci., 70, 3198-3215, https://doi.org/ 10.1175/JAS-D-12-0157.1.

Delpey, M. T., F. Ardhuin, F. Collard, and B. Chapron, 2010: Space-time structure of long ocean swell fields. J. Geophys. Res., 115, C12037, https://doi.org/10.1029/2009JC005885. 
Donelan, M. A., 2001: A nonlinear dissipation function due to wave breaking. Proc. ECMWF Workshop on Ocean Wave Forecasting, Reading, United Kingdom, ECMWF, 87-94.

- 2017: Frequency-direction spectra of wind-generated gravity waves. Encyclopedia of Maritime and Offshore Engineering, J. Carlton, P. Jukes, and Y. S. Choo, Eds., John Wiley and Sons, https://doi.org/10.1002/9781118476406.emoe079.

_ - J. Hamilton, and W. H. Hui, 1985: Directional spectra of wind-generated waves. Philos. Trans. Roy. Soc., 315A, 509562, https://doi.org/10.1098/rsta.1985.0054.

—-, A. V. Babanin, I. R. Young, M. L. Banner, and C. McCormick, 2005: Wave-follower field measurements of the wind-input spectral function. Part I: Measurements and calibrations. J. Atmos. Oceanic Technol., 22, 799-813, https:// doi.org/10.1175/JTECH1725.1.

,,--- , and $\longrightarrow$, 2006: Wave-follower field measurements of the wind-input spectral function. Part II: Parameterization of the wind input. J. Phys. Oceanogr., 36, 1672-1689, https://doi.org/10.1175/JPO2933.1.

Ewans, K. C., 1998: Observations of the directional spectrum of fetch-limited waves. J. Phys. Oceanogr., 28, 495-512, https:// doi.org/10.1175/1520-0485(1998)028<0495:OOTDSO > 2.0.CO;2.

_ , and A. C. Kibblewhite, 1990: An examination of fetchlimited wave growth off the west coast of New Zealand by a comparison with the JONSWAP results. J. Phys. Oceanogr., 20, 1278-1296, https://doi.org/10.1175/1520-0485(1990)020<1278: AEOFLW $>2.0 . \mathrm{CO} ; 2$.

Fan, Y., I. Ginis, and T. Hara, 2009a: The effect of wind-wavecurrent interaction on air-sea momentum fluxes and ocean response in tropical cyclones. J. Phys. Oceanogr., 39, 10191034, https://doi.org/10.1175/2008JPO4066.1.

,,,--- C. W. Wright, and E. J. Walsh, 2009b: Numerical simulations and observations of surface wave fields under an extreme tropical cyclone. J. Phys. Oceanogr., 39, 2097-2116, https://doi.org/10.1175/2009JPO4224.1.

Forristall, G. Z., 1981: Measurements of a saturated range in ocean wave spectra. J. Geophys. Res., 86, 8075-8084, https://doi.org/ 10.1029/JC086iC09p08075.

Gramstad, O., and A. V. Babanin, 2016: The generalized kinetic equation as a model for the nonlinear transfer in thirdgeneration wave models. Ocean Dyn., 66, 509-526, https:// doi.org/10.1007/s10236-016-0940-4.

Hanley, K. E., S. E. Belcher, and P. P. Sullivan, 2010: A global climatology of wind-wave interaction. J. Phys. Oceanogr., 40, 1263-1282, https://doi.org/10.1175/2010JPO4377.1.

Hanson, J. L., and O. M. Phillips, 2001: Automated analysis of ocean surface directional wave spectra. J. Atmos. Oceanic Technol., 18, 277-293, https://doi.org/10.1175/1520-0426(2001) $018<0277$ :AAOOSD > 2.0.CO;2.

Hasselmann, K., 1962: On the non-linear energy transfer in a gravity-wave spectrum: Part 1. General theory. J. Fluid Mech., 12, 481-500, https://doi.org/10.1017/S0022112062000373.

_ 1963: On the non-linear energy transfer in a gravity-wave spectrum: Part 3. Evaluation of the energy flux and swellsea interaction for a Neumann spectrum. J. Fluid Mech., 15, 385-398, https://doi.org/10.1017/S002211206300032X.

_ , and Coauthors, 1973: Measurements of wind-wave growth and swell decay during the Joint North Sea Wave Project (JONSWAP). Tech. Rep., Deutches Hydrographisches Institut, $93 \mathrm{pp}$.

— W. Sell, D. B. Ross, and P. Müller, 1976: A parametric wave prediction model. J. Phys. Oceanogr., 6, 200-228, https://doi.org/ 10.1175/1520-0485(1976)006<0200:APWPM>2.0.CO;2.
Hasselmann, S., and K. Hasselmann, 1985: Computations and parameterizations of the nonlinear energy transfer in a gravity-wave spectrum. Part I: A new method for efficient computations of the exact nonlinear transfer integral. J. Phys. Oceanogr., 15, 1369-1377, https://doi.org/10.1175/1520-0485(1985) 015<1369:CAPOTN $>2.0 . C O ; 2$.

,-- J. H. Allender, and T. P. Barnett, 1985: Computations and parameterizations of the nonlinear energy transfer in a gravity-wave spectrum. Part II: Parameterizations of the nonlinear energy transfer for application in wave models. J. Phys. Oceanogr., 15, 1378-1392, https://doi.org/10.1175/ 1520-0485(1985)015<1378:CAPOTN>2.0.CO;2.

— , and Coauthors, 1988: The WAM model-A third generation ocean wave prediction model. J. Phys. Oceanogr., 18, 1775-1810, https://doi.org/10.1175/1520-0485(1988)018<1775: TWMTGO > 2.0.CO;2.

Holthuijsen, L. H., 2007: Waves in Oceanic and Coastal Waters. Cambridge University Press, 404 pp., https://doi.org/10.1017/ CBO9780511618536.

Hwang, P. A., 2011: A note on the ocean surface roughness spectrum. J. Atmos. Oceanic Technol., 28, 436-443, https://doi.org/ 10.1175/2010JTECHO812.1.

— square slopes in the equilibrium and saturation ranges of the wave spectrum. J. Phys. Oceanogr., 31, 1346-1360, https://doi.org/ 10.1175/1520-0485(2001)031<1346:DDAMSS > 2.0.CO;2.

,-- E. J. Walsh, W. B. Krabill, and R. N. Swift, 2000a: Airborne measurements of the wavenumber spectra of ocean surface waves. Part I: Spectral slope and dimensionless spectral coefficient. J. Phys. Oceanogr., 30, 2753-2767, https:// doi.org/10.1175/1520-0485(2001)031<2753:AMOTWS > 2.0.CO;2. ,,,,---- and,$- 2000 \mathrm{~b}$ : Airborne measurements of the wavenumber spectra of ocean surface waves. Part II: Directional distribution. J. Phys. Oceanogr., 30, 2768-2787, https://doi.org/10.1175/1520-0485(2001)031<2768:AMOTWS> 2.0.CO;2.

Janssen, P. A. E. M., 2003: Nonlinear four-wave interactions and freak waves. J. Phys. Oceanogr., 33, 863-884, https://doi.org/ 10.1175/1520-0485(2003)33<863:NFIAFW >2.0.CO;2.

-_, 2004: The Interaction of Ocean Waves and Wind. Cambridge University Press, 312 pp., https://doi.org/10.1017/ CBO9780511525018.

Kahma, K. K., and C. J. Calkoen, 1992: Reconciling discrepancies in the observed growth of wind-generated waves. J. Phys. Oceanogr., 22, 1389-1405, https://doi.org/10.1175/ 1520-0485(1992)022<1389:RDITOG > 2.0.CO;2.

Komatsu, K., and A. Masuda, 1996: A new scheme of nonlinear energy transfer among wind waves: RIAM method-algorithm and performance. J. Oceanogr., 52, 509-537, https://doi.org/ 10.1007/BF02239052.

Komen, G. J., S. Hasselmann, and K. Hasselmann, 1984: On the existence of a fully developed wind-sea spectrum. J. Phys. Oceanogr., 14, 1271-1285, https://doi.org/10.1175/ 1520-0485(1984)014<1271:OTEOAF >2.0.CO;2.

_ L. Cavaleri, M. A. Donelan, K. Hasselmann, S. Hasselmann, and P. A. E. M. Janssen, 1994: Dynamics and Modelling of Ocean Waves. Cambridge University Press, 556 pp., https:// doi.org/10.1017/CBO9780511628955.

Krivinskii, B. B., 1991: Variability of energetic characteristics of wind surface waves (in Russian). Ph.D. thesis, Marine Hydrophysical Institute, Ukrainian National Academy of Sciences, $191 \mathrm{pp}$.

Kuik, A. J., G. P. van Vledder, and L. H. Holthuijsen, 1988: A method for the routine analysis of pitch-and-roll buoy wave 
data. J. Phys. Oceanogr., 18, 1020-1034, https://doi.org/10.1175/ 1520-0485(1988)018<1020:AMFTRA > 2.0.CO;2.

Lenain, L., and W. K. Melville, 2017: Measurements of the directional spectrum across the equilibrium-saturation ranges of wind-generated surface waves. J. Phys. Oceanogr., 47, 21232138, https://doi.org/10.1175/JPO-D-17-0017.1.

Lewis, A., and R. Allos, 1990: JONSWAP's parameters: Sorting out the inconsistencies. Ocean Eng., 17, 409-415, https:// doi.org/10.1016/0029-8018(90)90032-2.

Li, J.-G., 2012: Propagation of ocean surface waves on a spherical multiple-cell grid. J. Comput. Phys., 231, 8262-8277, https:// doi.org/10.1016/j.jcp.2012.08.007.

— , and M. Holt, 2009: Comparison of Envisat ASAR ocean wave spectra with buoy and altimeter data via a wave model. J. Atmos. Oceanic Technol., 26, 593-614, https://doi.org/ 10.1175/2008JTECHO529.1.

Liu, Q., A. V. Babanin, Y. Fan, S. Zieger, C. Guan, and I.-J. Moon, 2017: Numerical simulations of ocean surface waves under hurricane conditions: Assessment of existing model performance. Ocean Modell., 118, 73-93, https://doi.org/10.1016/j.ocemod.2017.08.005.

Manasseh, R., A. V. Babanin, C. Forbes, K. Rickards, I. Bobevski, and A. Ooi, 2006: Passive acoustic determination of wave-breaking events and their severity across the spectrum. J. Atmos. Oceanic Technol., 23, 599-618, https://doi.org/10.1175/JTECH1853.1.

Nose, T., A. V. Babanin, and K. C. Ewans, 2017: In situ observations of infragravity wave directionality at nearshore coastal sites. Ocean Sci. Discuss., https://doi.org/10.5194/os-2017-77.

Onorato, M., A. R. Osborne, M. Serio, L. Cavaleri, C. Brandini, and C. T. Stansberg, 2006: Extreme waves, modulational instability and second order theory: Wave flume experiments on irregular waves. Eur. J. Mech., 25B, 586-601, https://doi.org/ 10.1016/j.euromechflu.2006.01.002.

Perrie, W., B. Toulany, D. T. Resio, A. Roland, and J. P. Auclair, 2013: A two-scale approximation for wave-wave interactions in an operational wave model. Ocean Modell., 70, 38-51, https://doi.org/10.1016/j.ocemod.2013.06.008.

— with modern wave forecast models. J. Geophys. Res. Oceans, 123, 533-557, https://doi.org/10.1002/2017JC012868.

Pettersson, H., and K. Kahma, 2005: Directional measurements of wave growth in a short and narrow fetch geometry. J. Atmos. Ocean Sci., 10, 15-29, https://doi.org/10.1080/17417530500062853.

Phillips, O. M., 1958: The equilibrium range in the spectrum of wind-generated waves. J. Fluid Mech., 4, 426-434, https:// doi.org/10.1017/S0022112058000550.

_ 1984: On the response of short ocean wave components at a fixed wavenumber to ocean current variations. J. Phys. Oceanogr., 14, 1425-1433, https://doi.org/10.1175/1520-0485(1984) 014<1425:OTROSO >2.0.CO;2.

- 1985: Spectral and statistical properties of the equilibrium range in wind-generated gravity waves. J. Fluid Mech., 156, 505-531, https://doi.org/10.1017/S0022112085002221.

Portilla, J., F. J. Ocampo-Torres, and J. Monbaliu, 2009: Spectral partitioning and identification of wind sea and swell. J. Atmos. Oceanic Technol., 26, 107-122, https://doi.org/10.1175/ 2008JTECHO609.1.

Rapizo, H., T. Waseda, A. V. Babanin, and A. Toffoli, 2016: Laboratory experiments on the effects of a variable current field on the spectral geometry of water waves. J. Phys. Oceanogr., 46, 2695-2717, https://doi.org/10.1175/JPO-D-16-0011.1. , A. V. Babanin, D. Provis, and W. E. Rogers, 2017: Currentinduced dissipation in spectral wave models. J. Geophys. Res. Oceans, 122, 2205-2225, https://doi.org/10.1002/2016JC012367.
T. H. Durrant, and A. V. Babanin, 2018: An assessment of the impact of surface currents on wave modeling in the Southern Ocean. Ocean Dyn., 68, 939-955, https://doi.org/10.1007/ s10236-018-1171-7.

Rascle, N., and F. Ardhuin, 2013: A global wave parameter database for geophysical applications. Part 2: Model validation with improved source term parameterization. Ocean Modell., 70, 174-188, https://doi.org/10.1016/j.ocemod.2012.12.001.

Resio, D. T., and W. Perrie, 1991: A numerical study of nonlinear energy fluxes due to wave-wave interactions. Part 1: Methodology and basic results. J. Fluid Mech., 223, 603-629, https:// doi.org/10.1017/S002211209100157X.

_ , and — , 2008: A two-scale approximation for efficient representation of nonlinear energy transfers in a wind wave spectrum. Part I: Theoretical development. J. Phys. Ocean ogr., 38, 2801-2816, https://doi.org/10.1175/2008JPO3713.1.

— C. E. Long, and C. L. Vincent, 2004: Equilibrium-range constant in wind-generated wave spectra. J. Geophys. Res. 109, C01018, https://doi.org/10.1029/2003JC001788.

_ L. L. Vincent, and D. Ardag, 2016: Characteristics of directional wave spectra and implications for detailed-balance wave modeling. Ocean Modell., 103, 38-52, https://doi.org/10.1016/ j.ocemod.2015.09.009.

Ribal, A., A. V. Babanin, I. R. Young, A. Toffoli, and M. Stiassnie, 2013: Recurrent solutions of the Alber equation initialized by Joint North Sea Wave Project spectra. J. Fluid Mech., 719, 314-344, https://doi.org/10.1017/jfm.2013.7.

Rogers, W. E., 2017: Mean square slope in SWAN and WAVEWATCH III; buoy response functions; and limitations of ST1 physics in SWAN. Waves in Shallow Water Environments Meeting (WISE-24), Victoria, BC, Canada, https://www7320. nrlssc.navy.mil/dynamic/posters/rogers.afterWISE2017.pdf.

— , and D. W. Wang, 2006: On validation of directional wave predictions: Review and discussion. Tech. Rep., Naval Research Laboratory, 38 pp., https://apps.dtic.mil/dtic/tr/fulltext/ u2/a455737.pdf.

— , and - 2007: Directional validation of wave predictions. J. Atmos. Oceanic Technol., 24, 504-520, https://doi.org/10.1175/ JTECH1990.1.

_ - and G. P. van Vledder, 2013: Frequency width in predictions of windsea spectra and the role of the nonlinear solver. Ocean Modell., 70, 52-61, https://doi.org/10.1016/j.ocemod.2012.11.010.

— , P. A. Hwang, and D. W. Wang, 2003: Investigation of wave growth and decay in the SWAN model: Three regional-scale applications. J. Phys. Oceanogr., 33, 366-389, https://doi.org/ 10.1175/1520-0485(2003)033<0366:IOWGAD>2.0.CO;2.

—- A. V. Babanin, and D. W. Wang, 2012: Observationconsistent input and whitecapping dissipation in a model for wind-generated surface waves: Description and simple calculations. J. Atmos. Oceanic Technol., 29, 1329-1346, https://doi.org/10.1175/JTECH-D-11-00092.1.

Romero, L., and W. K. Melville, 2010a: Airborne observations of fetch-limited waves in the Gulf of Tehuantepec. J. Phys. Oceanogr., 40, 441-465, https://doi.org/10.1175/2009JPO4127.1.

_ and $-2010 \mathrm{~b}$ : Numerical modeling of fetch-limited waves in the Gulf of Tehuantepec. J. Phys. Oceanogr., 40, 466-486, https://doi.org/10.1175/2009JPO4128.1.

_, L. Lenain, and W. K. Melville, 2017: Observations of surface wave-current interaction. J. Phys. Oceanogr., 47, 615-632, https://doi.org/10.1175/JPO-D-16-0108.1.

Saha, S., and Coauthors, 2014: The NCEP Climate Forecast System version 2. J. Climate, 27, 2185-2208, https://doi.org/10.1175/ JCLI-D-12-00823.1. 
Stopa, J. E., F. Ardhuin, A. Babanin, and S. Zieger, 2016: Comparison and validation of physical wave parameterizations in spectral wave models. Ocean Modell., 103, 2-17, https:// doi.org/10.1016/j.ocemod.2015.09.003.

SWAN Team, 2018: SWAN Scientific and technical documentation: SWAN cycle III version 41.20AB. Delft University of Technology Rep., 139 pp., http://swanmodel.sourceforge.net/ download/zip/swantech.pdf.

Tamura, H., T. Waseda, Y. Miyazawa, and K. Komatsu, 2008: Current-induced modulation of the ocean wave spectrum and the role of nonlinear energy transfer. J. Phys. Oceanogr., 38, 2662-2684, https://doi.org/10.1175/2008JPO4000.1.

Taylor, K. E., 2001: Summarizing multiple aspects of model performance in a single diagram. J. Geophys. Res., 106, 71837192, https://doi.org/10.1029/2000JD900719.

Teixeira, M. A., and S. E. Belcher, 2002: On the distortion of turbulence by a progressive surface wave. J. Fluid Mech., 458 , 229-267, https://doi.org/10.1017/S0022112002007838.

Toba, Y., 1973: Local balance in the air-sea boundary processes. III. On the spectrum of wind waves. J. Oceanogr. Soc. Japan, 29, 209-220, https://doi.org/10.1007/BF02108528.

Tolman, H. L., 1991: A third-generation model for wind waves on slowly varying, unsteady, and inhomogeneous depths and currents. J. Phys. Oceanogr., 21, 782-797, https://doi.org/10.1175/ 1520-0485(1991)021<0782:ATGMFW>2.0.CO;2.

— 1992: Effects of numerics on the physics in a third-generation wind-wave model. J. Phys. Oceanogr., 22, 1095-1111, https:// doi.org/10.1175/1520-0485(1992)022<1095:EONOTP >2.0.CO;2. , 2003: Treatment of unresolved islands and ice in wind wave models. Ocean Modell., 5, 219-231, https://doi.org/10.1016/ S1463-5003(02)00040-9.

, 2010: Optimum discrete interaction approximations for wind waves. Part 4: Parameter optimization. Tech. Rep. 288, Environmental Modeling Center, Marine Modeling and Analysis Branch, 175 pp., http://polar.ncep.noaa.gov/mmab/papers/ tn288/MMAB_288.pdf.

_ 2011: A conservative nonlinear filter for the high-frequency range of wind wave spectra. Ocean Modell., 39, 291-300, https://doi.org/10.1016/j.ocemod.2011.05.004

_ 2013: A generalized multiple discrete interaction approximation for resonant four-wave interactions in wind wave models. Ocean Modell., 70, 11-24, https://doi.org/10.1016/j.ocemod.2013.02.005.

— wind wave model. J. Phys. Oceanogr., 26, 2497-2518, https:// doi.org/10.1175/1520-0485(1996)026<2497:STIATG >2.0.CO;2. , and R. W. Grumbine, 2013: Holistic genetic optimization of a generalized multiple discrete interaction approximation for wind waves. Ocean Modell., 70, 25-37, https://doi.org/10.1016/ j.ocemod.2012.12.008

Tracy, B., and D. T. Resio, 1982: Theory and calculation of the nonlinear energy transfer between sea waves in deep water. WES Rep. 11, U.S. Army Corps of Engineers, 56 pp., http:// www.dtic.mil/dtic/tr/fulltext/u2/a117989.pdf.

Tsagareli, K. N., A. V. Babanin, D. J. Walker, and I. R. Young, 2010: Numerical investigation of spectral evolution of wind waves. Part I: Wind-input source function. J. Phys. Oceanogr., 40, 656-666, https://doi.org/10.1175/2009JPO4345.1.

van der Westhuysen, A. J., M. Zijlema, and J. A. Battjes, 2007: Nonlinear saturation-based whitecapping dissipation in SWAN for deep and shallow water. Coastal Eng., 54, 151-170, https://doi.org/10.1016/j.coastaleng.2006.08.006.

van Vledder, G. P., 2006: The WRT method for the computation of non-linear four-wave interactions in discrete spectral wave models. Coastal Eng., 53, 223-242, https://doi.org/ 10.1016/j.coastaleng.2005.10.011.

S. T. Hulst, and J. D. McConochie, 2016: Source term balance in a severe storm in the southern North Sea. Ocean Dyn., 66, 1681-1697, https://doi.org/10.1007/s10236-016-0998-z.

Wang, D. W., and P. A. Hwang, 2001: Evolution of the bimodal directional distribution of ocean waves. J. Phys. Oceanogr., 31, 1200-1221, https://doi.org/10.1175/1520-0485(2001)031<1200: EOTBDD $>2.0 . \mathrm{CO} ; 2$.

Warner, J. C., B. Armstrong, R. He, and J. B. Zambon, 2010: Development of a Coupled Ocean-Atmosphere-Wave-Sediment Transport (COAWST) modeling system. Ocean Modell., 35, 230-244, https://doi.org/10.1016/j.ocemod.2010.07.010.

WAVEWATCH III Development Group, 2016: User manual and system documentation of WAVEWATCH III version 5.16. Environmental Modeling Center, Marine Modeling and Analysis Branch, NCEP, 360 pp., http://polar.ncep.noaa.gov/waves/ wavewatch/manual.v5.16.pdf.

Webb, D. J., 1978: Non-linear transfers between sea waves. Deep-Sea Res., 25, 279-298, https://doi.org/10.1016/0146-6291(78)90593-3.

Xiao, W., Y. Liu, G. Wu, and D. K. Yue, 2013: Rogue wave occurrence and dynamics by direct simulations of nonlinear wavefield evolution. J. Fluid Mech., 720, 357-392, https://doi.org/ 10.1017/jfm.2013.37.

Young, I. R., 1999: Wind Generated Ocean Waves. Elsevier Science Ltd., 287 pp.

2006: Directional spectra of hurricane wind waves. J. Geophys. Res., 111, C08020, https://doi.org/10.1029/2006JC003540. , and G. P. van Vledder, 1993: A review of the central role of nonlinear interactions in wind-wave evolution. Philos. Trans. Roy. Soc. London, 342A, 505-524, https://doi.org/10.1098/rsta.1993.0030. and A. V. Babanin, 2006: Spectral distribution of energy dissipation of wind-generated waves due to dominant wave breaking. J. Phys. Oceanogr., 36, 376-394, https://doi.org/10.1175/JPO2859.1.

, S. Hasselmann, and K. Hasselmann, 1987: Computations of the response of a wave spectrum to a sudden change in wind direction. J. Phys. Oceanogr., 17, 1317-1338, https://doi.org/ 10.1175/1520-0485(1987)017<1317:COTROA > 2.0.CO;2.

_ L. A. Verhagen, and M. L. Banner, 1995: A note on the bimodal directional spreading of fetch-limited wind waves. J. Geophys. Res., 100, 773-778, https://doi.org/10.1029/94JC02218.

_, M. L. Banner, M. A. Donelan, C. McCormick, A. V. Babanin, W. K. Melville, and F. Veron, 2005: An integrated system for the study of wind-wave source terms in finite-depth water. J. Atmos. Oceanic Technol., 22, 814-831, https://doi.org/10.1175/ JTECH1726.1.

, A. V. Babanin, and S. Zieger, 2013: The decay rate of ocean swell observed by altimeter. J. Phys. Oceanogr., 43, 2322-2333, https://doi.org/10.1175/JPO-D-13-083.1.

— E. Sanina, and A. V. Babanin, 2017: Calibration and cross validation of a global wind and wave database of altimeter, radiometer, and scatterometer measurements. J. Atmos. Oceanic Technol., 34, 1285-1306, https://doi.org/10.1175/ JTECH-D-16-0145.1.

Zakharov, V. E., 1968: Stability of periodic waves of finite amplitude on the surface of a deep fluid. J. Appl. Mech. Tech. Phys., 9, 190-194, https://doi.org/10.1007/BF00913182.

, 2018: Analytic theory of a wind-driven sea. Procedia IUTAM, 26, 43-58, https://doi.org/10.1016/j.piutam.2018.03.005.

Zieger, S., A. V. Babanin, W. E. Rogers, and I. R. Young, 2015: Observation-based source terms in the third-generation wave model WAVEWATCH. Ocean Modell., 96, 2-25, https://doi.org/10.1016/j.ocemod.2015.07.014. 